Document downloaded from:

http://hdl.handle.net/10251/50797

This paper must be cited as:

Reig, J. (2006). Capacity Analysis in Downlink WCDMA Systems Using Soft Handover Techniques With SIR-Based Power Control and Site Selection Diversity Transmission. IEEE Transactions on Vehicular Technology. 55(4):1362-1372. doi:10.1109/TVT.2006.877705.

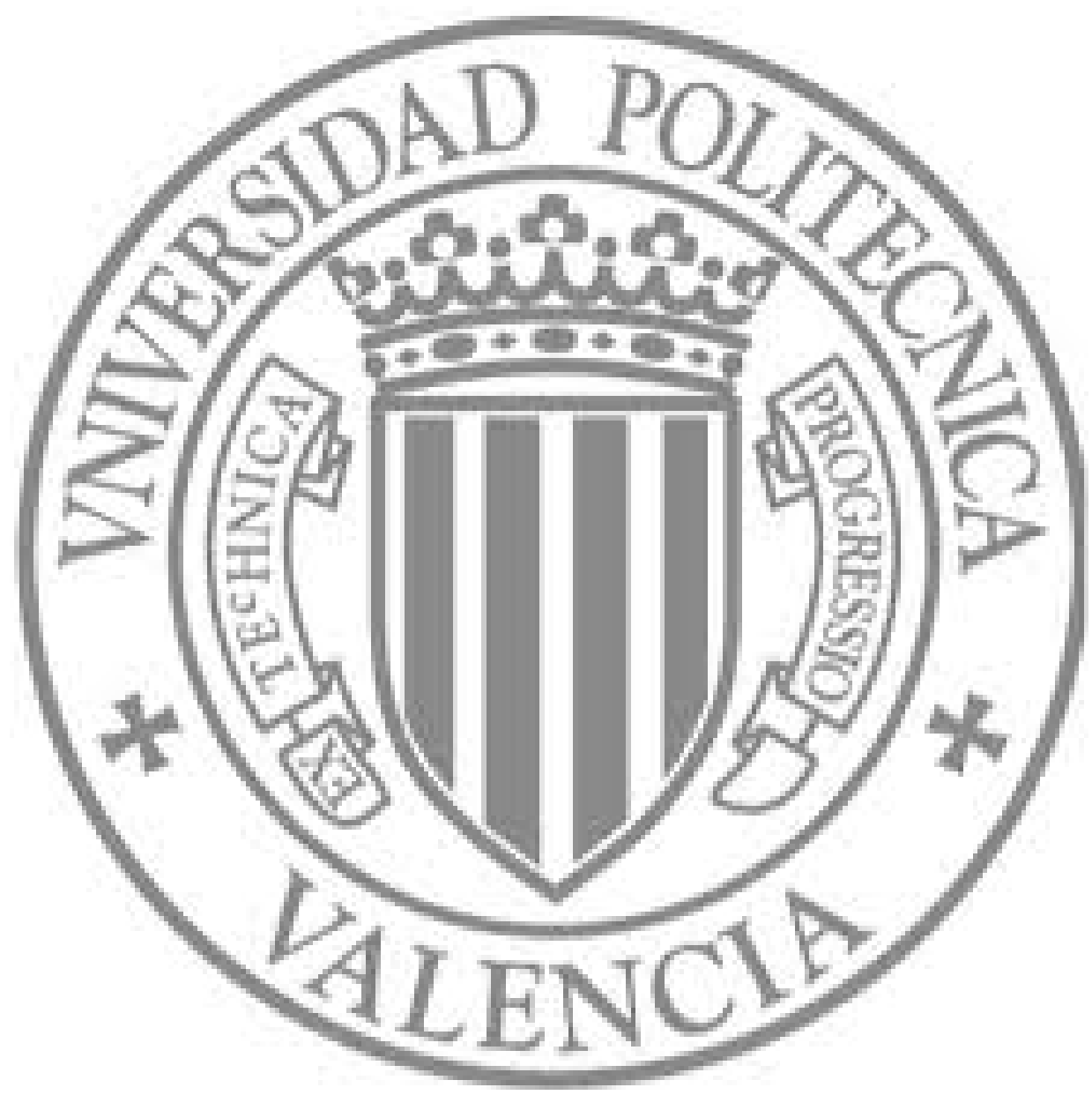

The final publication is available at

http://dx.doi.org/ 10.1109/TVT.2006.877705

Copyright Institute of Electrical and Electronics Engineers (IEEE) 


\title{
Capacity Analysis in Downlink WCDMA Systems using Soft Handover Techniques with SIR-based Power Control and Site Selection Diversity Transmission
}

\author{
Juan Reig, Member, IEEE
}

Manuscript received October 9, 2003. This work was supported in part by Motorola ANDC Spain.

J. Reig is with the Institute of Telecommunications and Multimedia Applications (ITEAM), Communications Department, Technical University of Valencia, 46022 Valencia SPAIN (e-mail: jreigp@dcom.upv.es). 


\title{
Capacity Analysis in Downlink WCDMA Systems using Soft Handover Techniques with SIR-based Power Control and Site Selection Diversity Transmission
}

\begin{abstract}
This work analyzes the downlink performance of a WCDMA system with site selection diversity transmission power control (SSDT) during soft handover mode. Signal to interference ratio (SIR) power control techniques are modeled and used in the simulations of this analysis. The study is focused on finding the optimum soft handover margin in terms of maximum system capacity under energy-per-bit to noise spectral density ratio $\left(E_{b} / N_{0}\right)$ quality requirements. The results of this analysis show an increase in user capacity of about $15-20 \%$ for optimum soft handover margins of $5-5.5 \mathrm{~dB}$. Nevertheless, the resources required (number of scrambling codes) by base station increase faster than the number of active users in terms of soft handover margin up to soft handover margin values of approximately $9.5 \mathrm{~dB}$.
\end{abstract}

Index Terms - Code division multiaccess, handover techniques, power control, soft handover margin

\section{INTRODUCTION}

Soft handover (SHO) is a technique that allows wireless user equipment to stay connected to several base stations (BS) in a WCDMA system. CDMA techniques make possible to maintain an old connection while adding a new one (make before break); however, additional resources from several BSs are required [1]. In downlink, this is achieved by multiple site transmission, which implies that several BSs transmit the same signal to a certain mobile station (MS). 
The capacity in CDMA systems is commonly limited by interference. In order to reduce the interference level in downlink, power control techniques are proposed in WCDMA systems. By using a power control algorithm that is based on signal to interference ratio (SIR) based power control in downlink, the power that is transmitted to the MS is adjusted to achieve the energy-per-bit to noise power spectral density ratio $\left(E_{b} / N_{0}\right)$ requirements [1], [2]. Therefore, more users can be served by the system if SIR-based power control techniques are used [2] since system interferences are reduced.

Two issues must be taken into account in SHO performance evaluation: the active set and the soft handover margin $\left(M_{S H}\right)$. The active set [3] is the group of base stations to which a user terminal is connected. $M_{S H}$ is the maximum allowed difference (measured in dB) between the power that is received from the best-server base station and the power that is received from a candidate base station that is included in the active set of the user terminal.

In $3^{\text {rd }}$ generation cellular systems, site selection diversity transmission power control (SSDT) is employed to mitigate interference produced by SHO multiple transmissions [4]: When using SSDT during SHO mode, only the best server BS is transmitting to the MS; the rest of BSs included in the active set turn off the power transmitted to this MS, maintaining only their dedicated physical control channel (DPCCH).

Previous works on the performance of uplink in WCDMA with soft handover in UMTS systems such as [5] show that SHO reduces interference, therefore SIR is increased. This effect is modeled as a SHO gain and can be used to offer higher quality services to users or to allocate a higher number of users in a cell. These analyses have demonstrated that capacity in uplink is always increased with $M_{S H}$.

In [6], Mehailescu et al. analyzed the downlink SHO performance in terms of $E_{b} / N_{0}$, assuming two candidate BSs in the active set. Connection probabilities were calculated to determine the connection situation of each MS location, but power control was not considered.

In the last few years, several authors have studied the effects of SHO on power-controlled downlink systems [7]-[9]. In [7], Yang et al. studied the effect of cross-correlated shadowing on the SIR using hard handover and soft handover algorithms. This work demonstrates that the 
constant cross-correlation model that is commonly used in the literature overestimates the SIR in WCDMA systems. In [8], Zhang et al. obtained the downlink capacity gain for SHO CDMA systems by dividing a hexagonal layout in defined connection zones. In [9], Chen and Cuthbert analyzed the performance of hard and soft handover algorithms in downlink WCDMA system using a probabilistic method. The mean active set number (the average number of base stations in the active set) is calculated in a mobility scenario where the environment model is limited to the path between two BSs.

Other authors have analyzed the downlink performance of different SHO algorithms (including SSDT technique) [10]-[15]. In [10], Staehle et al. compared SHO and SSDT techniques by evaluating the mean transmitted power by BS versus several parameters such as the mean of MS per BS, the orthogonality factor, etc. In [11], Heck et al. calculated the soft handover gain for SSDT within a WCDMA system; however, fading effects were not considered in [10] and [11]. In [12], Wang et al. obtained the capacity in terms of the number of users per cell for a certain outage probability without SHO. They also made a comparison between SHO and SSDT in a RAKE and generalized RAKE receiver. In [13], Akhtar et al. derived statistical distributions of BS transmitted power in SHO, SSDT and hybrid scheme for a certain soft handover margin (3dB) by simulation techniques. Other algorithms such as site independent diversity transmit power control (SIDTPC) and multiple site selection diversity transmit power control (M-SSDT) were proposed in [14] and [15], respectively, and compared to classical SSDT. In [16], Furukawa et al. evaluated the capacity in Kbps/MHz/sector using SSDT and assuming 5, 6, and infinity RAKE fingers. They showed that SSDT improves capacity from $27 \%$ to $57 \%$ for a pedestrian $\operatorname{MS}(4 \mathrm{Km} / \mathrm{h})$.

However, to the best of the author's knowledge, the optimum of soft handover for SSDT has not yet been analyzed in the literature. Therefore, this work evaluates the number of active users for several services or combined services in SSDT using a probabilistic scenario as a function of soft handover margin. This work also studies the influence on the optimum soft handover margin and the capacity of the propagation parameters, such as the constant of propagation, the shadowing standard deviation and the correlation coefficient between shadowing 
from each base station.

This paper is organized as follows. Section II contains a description of the system scenario modeling which includes an explanation of the selected area, the number of base stations considered, and the calculation of the connection probabilities in that area. Section III describes the downlink interference study which derives the SIR and $E_{b} / N_{0}$ equations. Section IV presents the capacity results obtained. Finally, our conclusions are addressed in section V.

\section{SCENARIO MODELING AND CONNECTION PROBABILITIES}

In this paper, a cellular hexagonal layout has been considered, where $\mathrm{BS}_{1}$ is the reference base station, that is interfered with by two tiers of base stations (18 BSs). The candidate base stations that are included in the active set are $\mathrm{BS}_{1}, \mathrm{BS}_{2}$, and $\mathrm{BS}_{3}$, as depicted in Fig. 1. We assume that a maximum of three BSs can be included in the active set.

In Fig. 1, the shadowed triangle represents the area under study. The results obtained for this area can be extended to the entire system area by using symmetry and rotation properties. To derive the average power transmitted from $\mathrm{BS}_{1}$, the triangle is meshed using a grid of $N$ points.

The signal received at the user terminal is affected by path loss and radio channel shadowing. In this paper, we only take into account shadowing, since fast fading is compensated by averaging in the user terminal. This shadowing effect is modeled as a lognormal distribution.

The probabilities of connecting the user terminals to the three candidate base stations must be calculated for every location in the triangle. The possible connection events at an $l$ location are: a single base station in the active set (with probabilities $P_{1, l}, P_{2, l}$, and $P_{3, l}$ corresponding to $\mathrm{BS}_{1}, \mathrm{BS}_{2}$ and $\mathrm{BS}_{3}$, respectively), $\mathrm{SHO}$ with two base stations ( $P_{12, l}$, and $P_{13, l}$,

with $\mathrm{BS}_{1}$ being the best server; $P_{21, l}$, and $P_{23, l}$, with $\mathrm{BS}_{2}$ being the best server; $P_{31, l}$, and $P_{32, l}$, with $\mathrm{BS}_{3}$ being the best server); and $\mathrm{SHO}$ with three base stations $\left(P_{123, i} ; P_{213, i} ; P_{312, l}\right)$.

To obtain these probabilities, we must calculate the received power signals measured in the common pilot channels $(\mathrm{CPICH})$ expressed in watts from $\mathrm{BS}_{1}, \mathrm{BS}_{2}$, and $\mathrm{BS}_{3}$ for an $l$ location in the area under study, assuming an omnidirectional radiation diagram for both BS and MS 
antennas. The received power signals are given by

$$
p_{r_{1, l}}=\frac{\varepsilon_{l} p_{p}}{d_{1, l}^{\mu}} \cdot 10^{\frac{\eta_{1, l}}{10}} \quad p_{r_{2, l}}=\frac{\varepsilon_{l} p_{p}}{d_{2, l}^{\mu}} \cdot 10^{\frac{\eta_{2, l}}{10}} \quad p_{r_{3, l}}=\frac{\varepsilon_{l} p_{p}}{d_{3, l}^{\mu}} \cdot 10^{\frac{\eta_{3, l}}{10}}
$$

The parameter $d_{j, l}, j=1,2,3$ is the distance from $\mathrm{BS}_{j}$ to MS, $\mu$ is the path loss exponent and $p_{p}$ is the CPICH power transmitted by BS in watt (which is assumed equal for every base station); the parameter $\eta_{j, l}, j=1,2,3$, is a Gaussian random variable with zero mean and standard deviation $\sigma$ (usually from 6 to $10 \mathrm{~dB}[17]$ ). This parameter represents the shadowing in the signal, as it propagates along the path from $\mathrm{BS}_{j}$ to MS. The parameter $\varepsilon_{l}$ is independent of $d_{j, l}$ and is given by

$$
\varepsilon_{l}=\frac{g_{t, l} \cdot g_{r}}{10^{\frac{L_{C}}{10}} \cdot 10^{\frac{A}{10}}} .
$$

The parameter $g_{t, l}$ is the BS antenna gain in the angle from BS to an $l$ location, which is assumed to be equal for all BS for a given angle. The parameter $g_{r}$ is the MS antenna gain and $L_{C}$ is the cable and conector losses in $\mathrm{dB}$. The parameter $A$ depends on the propagation model used (it includes the path loss term in $\mathrm{dB}$ independent of the distance between the transmitter and the receiver).

Since the shadowing in the received signal depends on the mobile station environment, the variables $\eta_{i, l}$ and $\eta_{j, l}(i, j=1,2,3, i \neq j)$ are correlated [18]. Therefore, a correlation coefficient between $\eta_{i, l}$ and $\eta_{j, l}, \rho$, defined as

$$
\rho=\frac{\operatorname{cov}\left(\eta_{i, l}, \eta_{j, l}\right)}{\sigma^{2}} \quad i, j=1,2,3, \quad i \neq j,
$$

will be used below, where $\operatorname{cov}\left(\eta_{i, l}, \eta_{j, l}\right)$ denotes the covariance between $\eta_{i, l}$ and $\eta_{j, l}$.

From (1), we can derive the best server $\mathrm{BS}_{j}$ at an $l$ position as

$$
P_{r_{j, l}}=\max \left(P_{r_{1, l}}, P_{r_{2, l}}, P_{r_{3, l}}\right)
$$

where $P_{r_{i, l}}(i=1,2,3)$ represent the received powers, $p_{r_{i, l}}(i=1,2,3)$, which are expressed in logarithmic units.

Therefore, the minimum received power signal required at an $l$ location to include a new base station in the active set is given by 


$$
P_{r_{\min }, l}=P_{r_{j, l}}-M_{S H}
$$

where $M_{S H}$ is the soft handover margin expressed in $\mathrm{dB}$.

Signals arriving from the $\mathrm{BS}_{i}(i=1,2,3)$ at an $l$ position whose power is higher than $P_{r_{\min }, l}$ are included in the active set of the user terminal.

Thus, the connection probabilities for the $l$ position in the triangle can be expressed as

$$
\begin{aligned}
& P_{1, l}=\operatorname{Prob}\left(P_{r_{1, l}}-P_{r_{2, l}}>M_{S H}, P_{r_{2, l}}-P_{r_{3, l}} \geq 0\right)+\operatorname{Prob}\left(P_{r_{1, l}}-P_{r_{3, l}}>M_{S H}, P_{r_{3, l}}-P_{r_{2, l}}>0\right) \\
& P_{2, l}=\operatorname{Prob}\left(P_{r_{2, l}}-P_{r_{1, l}}>M_{S H}, P_{r_{1, l}}-P_{r_{3, l}} \geq 0\right)+\operatorname{Prob}\left(P_{r_{2, l}}-P_{r_{3, l}}>M_{S H}, P_{r_{3, l}}-P_{r_{1, l}}>0\right) \\
& P_{3, l}=\operatorname{Prob}\left(P_{r_{3, l}}-P_{r_{1, l}}>M_{S H}, P_{r_{1, l}}-P_{r_{2, l}} \geq 0\right)+\operatorname{Prob}\left(P_{r_{3, l}}-P_{r_{2, l}}>M_{S H}, P_{r_{2, l}}-P_{r_{1, l}}>0\right) \\
& P_{12, l}=\operatorname{Prob}\left(0 \leq P_{r_{1, l}}-P_{r_{2, l}}<M_{S H}, P_{r_{1, l}}-P_{r_{3, l}}>M_{S H}\right) \\
& P_{21, l}=\operatorname{Prob}\left(0<P_{r_{2, l}}-P_{r_{1, l}}<M_{S H}, P_{r_{2, l}}-P_{r_{3, l}}>M_{S H}\right) \\
& P_{13, l}=\operatorname{Prob}\left(0 \leq P_{r_{1, l}}-P_{r_{3, l}}<M_{S H}, P_{r_{1, l}}-P_{r_{2, l}}>M_{S H}\right) \\
& P_{31, l}=\operatorname{Prob}\left(0<P_{r_{3, l}}-P_{r_{1, l}}<M_{S H}, P_{r_{3, l}}-P_{r_{2, l}}>M_{S H}\right) \\
& P_{23, l}=\operatorname{Prob}\left(0 \leq P_{r_{2, l}}-P_{r_{3, l}}<M_{S H}, P_{r_{2, l}}-P_{r_{1, l}}>M_{S H}\right) \\
& P_{32, l}=\operatorname{Prob}\left(0<P_{r_{3, l}}-P_{r_{2, l}}<M_{S H}, P_{r_{3, l}}-P_{r_{1, l}}>M_{S H}\right) \\
& P_{123, l}=\operatorname{Prob}\left(0 \leq P_{r_{1, l}}-P_{r_{2, l}}<M_{S H}, 0 \leq P_{r_{1, l}}-P_{r_{3, l}}<M_{S H}\right) \\
& P_{213, l}=\operatorname{Prob}\left(0<P_{r_{2, l}}-P_{r_{1, l}}<M_{S H}, 0 \leq P_{r_{2, l}}-P_{r_{3, l}}<M_{S H}\right) \\
& P_{312, l}=\operatorname{Prob}\left(0<P_{r_{3, l}}-P_{r_{1, l}}<M_{S H}, 0<P_{r_{3, l}}-P_{r_{2, l}}<M_{S H}\right),
\end{aligned}
$$

where $\operatorname{Prob}(\cdot)$ denotes probability.

The events involved in these probabilities are not independent, since the received power signal variables are implicated simultaneously in several events, and the received power signal variables have certain correlations. Since $P_{r_{1}, 1}, P_{r_{2,1}}$, and $P_{r_{3,1}}$ are assumed to be Gaussian distributed, the subtraction of two of them follows a Gaussian distribution [19], whose standard deviation is given by

$$
\sigma_{e q}=\sigma \cdot \sqrt{2(1-\rho)}
$$

Using the bivariate normal distribution, the probabilities (6)-(12) can be calculated as shown in the appendix.

An example of the connection probabilities in the triangle (Fig. 1) for $M_{S H}=4 \mathrm{~dB}$ is shown in Fig. 2. These probability values decrease as $M_{S H}$ increases, and the soft handover areas 
increase as $M_{S H}$ increases. Note that the sum of all the probabilities in every location of the triangle is equal to 1 (see Fig. 2).

As Fig. 2 shows, the single base station connection probabilities, $P_{1, l}, P_{2, l}$, and $P_{3, l}$, are symmetrically circular around the corresponding base station. Their value ranges from 1 (at the base station position) to zero (at a certain distance from the base station position).

Thus, the areas and values of the connection probabilities depend strongly on the $M_{S H}$ parameter value. Fig. 3 shows the percentage of users in the cell connected to one, $\left(N_{1}\right)$, two $\left(N_{2}\right)$, or three $\left(N_{3}\right)$ base stations simultaneously, given by

$$
\begin{gathered}
N_{1}=\frac{\sum_{l=1}^{N} P_{1, l}}{\sum_{l=1}^{N}\left(P_{1, l}+P_{12, l}+P_{13, l}+P_{123, l}\right)}, \\
N_{2}=\frac{\sum_{l=1}^{N}\left(P_{12, l}+P_{13, l}\right)}{\sum_{l=1}^{N}\left(P_{1, l}+P_{12, l}+P_{13, l}+P_{123, l}\right)}, \\
N_{3}=\frac{\sum_{l=1}^{N} P_{123, l}}{\sum_{l=1}^{N}\left(P_{1, l}+P_{12, l}+P_{13, l}+P_{123, l}\right)} .
\end{gathered}
$$

Note that if $M_{S H}$ is $0 \mathrm{~dB}$, the percentage of users in handover is zero.

\section{DOWNLINK INTERFERENCE ANALYSIS.}

Since SIR power control must be simulated, interference modelling is required in this work. For the proposed scenario, the SIR value at each MS position in the area is calculated and fixed to its target value by modifying the corresponding dedicated physical data channel (DPDCH)-BS transmitted power.

The interferences in the cellular system can be divided into two types [20]: intracell $\left(I_{\text {intra }}\right)$ and intercell $\left(I_{\text {inter }}\right)$. Both kinds of interference can be used to obtain the $E_{b} / N_{0}$ relationship to derive the required transmission power to satisfy the quality requirements. Fig. 4 shows these kinds of interference in a WCDMA system. 
Let $\gamma_{i, l}$ be the $E_{b} / N_{0}$ measured in the mobile station at an $l$ location from $\operatorname{BS}_{i}(i=1,2,3)$ given by

$$
\gamma_{i, l}=G_{P} \frac{C_{i, l}}{I_{\text {inter }_{i, l}}+I_{\text {intra }_{i, l}}+N_{0}}=G_{P}\left(\frac{I_{\text {inter }_{i, l}}}{C_{i, l}}+\frac{I_{\text {intra }_{i, l}}}{C_{i, l}}+\frac{N_{0}}{C_{i, l}}\right)^{-1} .
$$

The parameter $N_{0}$ is the receiver thermal noise power given by

$$
N_{0}=10 \log \left(k T B_{W}\right)+F,
$$

where $k$ is the Boltzmann constant $\left(1.38 \cdot 10^{-23} \mathrm{~J} / \mathrm{K}\right), T$ is the noise temperature of the antenna $(290 \mathrm{~K}), B_{W}$ is the MS bandwidth, $F$ is the noise figure of the receiver, and $C_{i, l}$ is the power signal received from $\mathrm{BS}_{i}(i=1,2,3)$ by the MS at an $l$ location for a given service, given by the following equation

$$
C_{i, l}=p_{i, l} \varepsilon_{l} d_{i, l}{ }^{-\mu} 10^{\eta_{i, l} / 10} .
$$

The parameter $p_{i, l}$ is the power transmitted by $\mathrm{BS}_{i}$ to the $l$ location study in the DPDCH, and $G_{p}$ is the process gain, defined as

$$
G_{P}=\frac{B}{R},
$$

where $B$ is the spread signal chip rate expressed in chips per second, and $R$ is the bearer signal bit rate in bits per second, which depends on the service bit rate.

If we take expectations in the second term of (17), as this term is a convex function, we can write Jensen's inequality for convex functions as follows

$$
\bar{\gamma}_{i, l} \geq G_{P}\left(E\left[\frac{I_{\text {inter }_{i, l}}}{C_{i, l}}\right]+E\left[\frac{I_{\mathrm{intra}_{i, l}}}{C_{i, l}}\right]+E\left(\frac{N_{0}}{C_{i, l}}\right)\right)^{-1},
$$

where $E[\cdot]$ denotes expectation.

In order to satisfy quality requirements, the equation (21) must satisfy the following condition at an $l$ position

$$
\bar{\gamma}_{i, l} \geq\left.\frac{E_{b}}{N_{0}}\right|_{r e q}
$$

where $E_{b} /\left.N_{0}\right|_{r e q}$ is the threshold energy-per-bit to noise power spectral density ratio in linear units for each service. 


\section{A. Intracell Interference}

The intracell interference received at a certain $l$ position of MS is produced by the power transmitted to other users in the same base station. Assuming identical total transmitted power for all BS, the total power transmitted by each base station $\operatorname{BS}_{i}(i=1,2,3)$ is given by

$$
p_{t}=p_{p}+\sum_{l=1}^{N} p_{i, l} \quad, i=1,2,3 .
$$

For a certain user at an $l$ location, all the power transmitted by the base station $\mathrm{BS}_{i}$ is considered as interfering except for $p_{i, l}$, which is the required power transmitted by $\mathrm{BS}_{i}$ to satisfy the $E_{b} / N_{0}$ target for this user.

The interference power received by MS at an $l$ location from its own $\mathrm{BS}_{i}$ can be written as

$$
I_{\text {intra }_{i, l}}=\left[p_{p}+\alpha \cdot \delta \sum_{j=1, j \neq l}^{N} p_{i, j}\right] \varepsilon_{l} d_{i, l}{ }^{-\mu} 10^{\eta_{i, l} / 10}=\left(p_{p l}+\alpha \cdot \delta\left(p_{t}-p_{p}-p_{i, l}\right)\right) \varepsilon_{l} d_{i, l}{ }^{-\mu} 10^{\eta_{i, l} / 10},
$$

where $\alpha$ is the orthogonality factor among spreading codes (considered 0.5 due to the multipath effect [21]), and where $\delta$ is the activity factor.

From (17) and (24) we can obtain

$$
\frac{I_{\text {intra }_{i, l}}}{C_{i, l}}=\frac{\alpha\left(p_{p}+\delta\left(p_{t}-p_{p}-p_{i, l}\right)\right)}{p_{i, l}}=\alpha \delta\left(\frac{p_{p}}{\delta p_{i, l}}+\frac{p_{t}-p_{p}}{p_{i, l}}-1\right) .
$$

\section{B. Intercell Interference}

Intercell interferences are those produced by transmissions from base stations that are not the base station that the mobile station is connected to. In order to calculate these interferences, we assume two different situations: the MS is connected to a single BS, i.e. it is not in soft handover (without macrodiversity), or the MS is connected to two or three base station simultaneously, i.e. it is in soft handover (with macrodiversity).

First, the interferences without macrodiversity are calculated. Since the user equipment is connected to only one base station (its best server), the signals received from other base stations are interferences. In this paper, we assume that the 18 interfering base stations $\mathrm{BS}_{j}$ are 
transmitting the same power $p_{t}$. The intercell interference power received by a MS at an $l$ position whose best server is $\mathrm{BS}_{i}$ is given by

$$
I_{\text {inter }_{, l}}=\delta \varepsilon_{l} \sum_{\substack{j=2 \\ j \neq i}}^{19} p_{t} d_{j, l}^{-\mu} 10^{\eta_{j, l} 110} .
$$

To take this condition into account, mathematical expectations are used. These expectations can be stated as the integral of the probability density function of the lognormal Gaussian random variable. Using the function $Q$, this integral can be evaluated as follows [6]

$$
\begin{aligned}
& E\left[\frac{I_{\text {inter }_{i, l}}}{C_{i, l}}\right]=\delta \frac{p_{t}}{p_{i, l}} \sum_{\substack{j=2 \\
j \neq i}}^{19}\left(\frac{d_{i, l}}{d_{j, l}}\right)^{\mu} E\left[10^{\frac{\eta_{j, l}-\eta_{i, l}}{10}}, P_{r_{i, l}}>P_{r_{j, l}}+M_{S H}\right]= \\
& \delta \frac{p_{t}}{p_{i, l}} e^{\left(a \sigma_{e q}\right)^{2}} \sum_{\substack{j=2 \\
j \neq i}}^{19}\left(\frac{d_{i, l}}{d_{j, l}}\right)^{\mu} Q\left(a \cdot \sigma_{e q}+\frac{\left.10 \mu \log \left(\frac{d_{i, l}}{d_{j, l}}\right)+M_{S H}\right)}{\sigma_{e q}}\right),
\end{aligned}
$$

where $a=\ln (10) / 10, i=1,2,3$ is the best server base station, and $Q$ is defined as $[22$, eq.(26.2.3)]

$$
Q(x)=\frac{1}{\sqrt{2 \pi}} \int_{x}^{\infty} e^{-z^{2} / 2} d z
$$

Using macrodiversity, the MS has several base stations in its active set; each of them is transmitting the desired signal, while the rest of the BS are considered interferences.

The following equation applies when base stations $\mathrm{BS}_{i}$ and $\mathrm{BS}_{j}$ are included in the active set, with $\mathrm{BS}_{i}$ being the best server

$$
\begin{aligned}
& E\left[\frac{I_{\text {inter }_{i, l}}}{C_{i, l}}\right]=\delta \frac{p_{t}}{p_{i, l}}\left(\frac{d_{i, l}}{d_{j, l}}\right)^{\mu} E\left[10^{\frac{d_{j, l}-d_{i, l}}{10}}, P_{r_{j, l}}-M_{S H}<P_{r_{i, l}}<P_{r_{j, l}}+M_{S H}\right]+ \\
& +\delta \frac{p_{t}}{p_{i, l}} \sum_{m=3}^{19}\left(\frac{d_{i, l}}{d_{m, l}}\right)^{\mu} E\left[10^{\frac{d_{m, l}-d_{i, l}}{10}}, P_{r_{i, l}}>P_{r_{m, l}}+M_{S H}\right]
\end{aligned}
$$

Using Q functions, (29) can be expanded as 


$$
\begin{aligned}
& E\left[\frac{I_{\text {inter } r_{i, l}}}{C_{i, l}}\right]=\delta \frac{p_{t}}{p_{i, l}}\left(\frac{d_{i, l}}{d_{j, l}}\right)^{\mu} e^{\left(a \sigma_{e q}\right)^{2}} Q\left(a \cdot \sigma_{e q}+\frac{10 \mu \log \left(\frac{d_{i, l}}{d_{j, l}}\right)-M_{S H}}{\sigma_{e q}}\right)- \\
& -\delta \frac{p_{t}}{p_{i, l}}\left(\frac{d_{i, l}}{d_{j, l}}\right)^{\mu} e^{\left(a \sigma_{e q}\right)^{2}} Q\left(\begin{array}{c}
10 \mu \log \left(\frac{d_{i, l}}{d_{j, l}}\right)+M_{S H} \\
\sigma_{e q}
\end{array}\right)+ \\
& +\delta \frac{p_{t}}{p_{i, l}} e^{\left(a \sigma_{e q}\right)^{2}} \sum_{m=3}^{19}\left(\frac{d_{i, l}}{d_{m, l}}\right)^{\mu} Q\left(\begin{array}{c}
10 \mu \log \left(\frac{d_{i, l}}{d_{m, l}}\right)+M_{S H} \\
\sigma_{e q}
\end{array}\right)
\end{aligned}
$$

with $i=1,2,3 ; j=1,2,3 ; i \neq j$, and where the best server BS is denoted in the first sub-index and the rest of BSs are denoted in the second one.

Analogously, we can obtain the expression for the case when the user at an $l$ location is connected to three base stations simultaneously, with $\mathrm{BS}_{i}$ being the best server and $\mathrm{BS}_{j}$ and $\mathrm{BS}_{q}$ in the active set, written as

$$
\begin{aligned}
& E\left[\frac{I_{\text {inter }}}{C_{i, l, l}}\right]=\delta \frac{p_{t}}{p_{i, l}}\left(\frac{d_{i, l}}{d_{j, l}}\right)^{\mu} E\left[10^{\frac{d_{j, l}-d_{i, l}}{10}}, P_{r_{j, l}}-M_{S H}<P_{r_{i, l}}<P_{r_{, j}}+M_{S H}\right]+ \\
& +\delta \frac{p_{t}}{p_{i, l}}\left(\frac{d_{i, l}}{d_{q, l}}\right)^{\mu} E\left[10^{\frac{d_{q, l}-d_{i, l}}{10},}, P_{r_{q, l}}-M_{S H}<P_{r_{i, l}}<P_{r_{q, l}}+M_{S H}\right]+ \\
& +\delta \frac{p_{t}}{p_{i, l}} \sum_{m=4}^{19}\left(\frac{d_{i, l}}{d_{m, l}}\right)^{\mu} E\left[10^{\frac{d_{m, l}-d_{i, l}}{10}}, P_{r_{i, l}}>P_{r_{m, l}}+M_{S H}\right]
\end{aligned}
$$

From (31), we can obtain 


$$
\begin{aligned}
& E\left[\frac{I_{\text {inter } \mathrm{r}_{j, l}, l}}{C_{i, l}}\right]=\delta \frac{p_{t}}{p_{i, l}}\left(\frac{d_{i, l}}{d_{j, l}}\right)^{\mu} e^{\left(a \sigma_{e q}\right)^{2}} Q\left(a \cdot \sigma_{e q}+\frac{10 \mu \log \left(\frac{d_{i, l}}{d_{j, l}}\right)-M_{S H}}{\sigma_{e q}}\right)- \\
& -\delta \frac{p_{t}}{p_{i, l}}\left(\frac{d_{i, l}}{d_{j, l}}\right)^{\mu} e^{\left(a \sigma_{e q}\right)^{2}} Q\left(a \cdot \sigma_{e q}+\frac{10 \mu \log \left(\frac{d_{i, l}}{d_{j, l}}\right)+M_{S H}}{\sigma_{e q}}\right)+ \\
& +\delta \frac{p_{t}}{p_{i, l}}\left(\frac{d_{i, l}}{d_{q, l}}\right)^{\mu} e^{\left(a \sigma_{e q}\right)^{2}} Q\left(a \cdot \sigma_{e q}+\frac{10 \mu \log \left(\frac{d_{i, l}}{d_{q, l}}\right)-M_{S H}}{\sigma_{e q}}\right)- \\
& -\delta \frac{p_{t}}{p_{i, l}}\left(\frac{d_{i, l}}{d_{q, l}}\right)^{\mu} e^{\left(a \sigma_{e q}\right)^{2}} Q\left(a \cdot \sigma_{e q}+\frac{10 \mu \log \left(\frac{d_{i, l}}{d_{q, l}}\right)+M_{S H}}{\sigma_{e q}}\right)+ \\
& +\delta \frac{p_{t}}{p_{i, l}} e^{\left(a \sigma_{e q}\right)^{2}} \sum_{m=4}^{19}\left(\frac{d_{i, l}}{d_{m, l}}\right)^{\mu} Q\left(a \cdot \sigma_{e q}+\frac{10 \mu \log \left(\frac{d_{i, l}}{d_{m, l}}\right)+M_{S H}}{\sigma_{e q}}\right)
\end{aligned}
$$

where $i=1,2,3 ; j=1,2,3 ; q=1,2,3 ; i \neq j \neq q$.

\section{Derivation of $E_{b} / N_{0}$}

Using (24) and (26), the $E_{b} / N_{0}$ for a MS at an $l$ location connected to a single base station $\mathrm{BS}_{i}$ (without macrodiversity) can be calculated from

$$
\bar{\gamma}_{i, l}=\frac{G_{P}}{E\left[\frac{I_{\text {intra }_{i, l}}}{C_{i, l}}\right]+E\left[\frac{I_{\text {inter }_{i, l}}}{C_{i, l}}\right]+\frac{N_{0}}{C_{i, l}}} .
$$

Considering macrodiversity, the intracell interference is similar; therefore, (24) can be used to obtain the $E_{b} / N_{0}$. The next equation denotes two base stations, $\mathrm{BS}_{i}$ and $\mathrm{BS}_{j}$, included in the active set at position $l$, with $\mathrm{BS}_{i}$ being the best serve

$$
\bar{\gamma}_{i j, l}=\frac{G_{P}}{E\left[\frac{I_{\text {ntra }_{i, l}}}{C_{i, l}}\right]+E\left[\frac{I_{\text {inter }_{i, l}}}{C_{i, l}}\right]+\frac{N_{0}}{C_{i, l}}},
$$

with $i=1,2,3 ; j=1,2,3 ; i \neq j$. 
We can also derive the $E_{b} / N_{0}$ when the user is connected to three base stations simultaneously as

$$
\bar{\gamma}_{i j q, l}=\frac{G_{p}}{E\left[\frac{I_{\text {intra }_{i, l}}}{C_{i, l}}\right]+E\left[\frac{I_{\text {inter }_{j, l}, l}}{C_{i, l}}\right]+\frac{N_{0}}{C_{i, l}}},
$$

with $i=1,2,3 ; j=1,2,3 ; q=1,2,3 ; i \neq j \neq q$.

For a certain location in the area under study, we cannot calculate in a deterministic way the base stations which the user terminal is connected to. It is not even possible to assure if the user terminal is in soft handover situation or not, due the random characteristic of the SIR in the receiver.

Assuming selection combining (SC) [4], $\bar{\gamma}_{\text {total, }}$ defined as the mean SIR received at an $l$ location, can be written as

$$
\begin{aligned}
& \bar{\gamma}_{\text {total, } l}=P_{1, l} \cdot \bar{\gamma}_{1, l}+P_{2, l} \cdot \bar{\gamma}_{2, l}+P_{3, l} \cdot \bar{\gamma}_{3, l}+P_{12, l} \cdot \bar{\gamma}_{12, l}+P_{21, l} \cdot \bar{\gamma}_{21, l}+P_{13, k} \cdot \bar{\gamma}_{13, l}+P_{31, l} \cdot \bar{\gamma}_{31, l}+ \\
& +P_{23, l} \cdot \bar{\gamma}_{23, l}+P_{32, l} \cdot \bar{\gamma}_{32, l}+P_{123, l} \cdot \bar{\gamma}_{123, l}+P_{213, l} \cdot \bar{\gamma}_{213, l}+P_{312, l} \cdot \bar{\gamma}_{312, l}
\end{aligned},
$$

using (34) and (35).

Using (21) and (36), we can obtain the minimum value for the transmitted power $p_{1, l}$ from $\mathrm{BS}_{1}$ to user at the $l$ location, by assuming $p_{1, l}=p_{2, l}=p_{3, l}$ for simplicity. From (36), the weighted average transmitted power in DPDCH over $N$ points of the triangle can be derived as

$$
\overline{p_{1}}=\frac{\sum_{l=1}^{N}\left(P_{1, l}+P_{12, l}+P_{13, l}+P_{123, l}\right) \cdot p_{1, l}}{\sum_{l=1}^{N}\left(P_{1, l}+P_{12, l}+P_{13, l}+P_{123, l}\right)} .
$$

In order to satisfy the quality requirements (22) in the overall area, the available power at base station $\mathrm{BS}_{1}\left(p_{t}\right)$ must be distributed among the users of the area under study whose best server is $\mathrm{BS}_{1}$. The average number of users that require power from $\mathrm{BS}_{1}$ yields the number of transmitted codes, i.e. the cell capacity, $n_{t r x}$ given by

$$
n_{t r x}=\frac{p_{t}}{\overline{p_{1}}}
$$

If we take into account that several users are connected to more than one BS, the number of required codes, $n_{\text {req }}$, can be obtained as

$$
n_{r e q}=n_{t r x}\left(1-N_{2}-N_{3}\right)+2 n_{t r x} N_{2}+3 n_{t r x} N_{3}
$$


where $N_{2}, N_{3}$, and $n_{t r x}$ are defined in (15), (16) and (38), respectively.

Therefore, the graphics obtained in this study consist of two types of curves: the capacity curve, which indicates the maximum number of active users served per base station, and the required codes curve, which indicates the required resources per base station to serve this number of users.

\section{NUMERICAL RESULTS}

The default parameter values used in our simulations are as follows

$B=3.84$ Mchip $/ \mathrm{s}$

$R=12200 \mathrm{~b} / \mathrm{s}$

$\rho=0.5$

$L(d B)=A+10 \mu \log d_{j, l}(K m)=$

$152.5+35.2 \log d_{j, l}(K m)$

$L_{C}=6 \mathrm{~dB}$

$\alpha=0.5$

$\delta=0.5$

$E_{b} /\left.N_{0}\right|_{r e q}=6.4 d B$

$\sigma=8 \mathrm{~dB}$

$p_{t}=20 \mathrm{w}$

$p_{p}=2 \mathrm{w}$

$B_{W}=5 \mathrm{MHz}$

$F=5 \mathrm{~dB}$

$D=400 \mathrm{~m}$

Uniform spread signal chip rate;

speech bit rate;

correlation coefficient;

path loss;

antenna cable losses;

orthogonality factor;

activity factor (for speech users);

threshold energy per bit to noise spectral density

(for speech users);

shadowing standard deviation;

total transmitted power;

transmitted power in pilot channel (CPICH);

noise equivalent bandwidth;

noise figure of receiver;

cell radius;

distribution of users;

Simulations for different types of services and environments were performed, using the equations obtained in the previous sections to model the connection probabilities in a soft 
handover scenario and to calculate the total amount of users and channel elements per cell. The number of points in the triangle of the simulation for each value of $M_{S H}$ was 1511 . The number of active users and the resources obtained using (38) and (39) converged from around 500 points in the triangle. The soft handover margin was ranged from $0 \mathrm{~dB}$ to $12 \mathrm{~dB}$ in intervals of $0.5 \mathrm{~dB}$.

In Fig. 5, the number of connected users and the cell capacity are plotted for voice service $(R=12.2 \mathrm{~Kb} / \mathrm{s}), B=3.84 \mathrm{Mchip} / \mathrm{s}, \sigma=8 \mathrm{~dB}, \mu=3.5, \rho=0.5$, and a cell radius of 400 m.

For this situation, the optimum $M_{S H}$ achieved was $5.5 \mathrm{~dB}$ and the capacity gain was 18.5 $\%$, while the required resources increase was $69 \%$.

The optimum $M_{S H}$ obtained was always about $5.5 \mathrm{~dB}$ for any class of service analyzed (voice, $144 \mathrm{~Kb} / \mathrm{s}$ data, $384 \mathrm{~Kb} / \mathrm{s}$ data and $2 \mathrm{Mb} / \mathrm{s}$ data). Table 1 contains the main parameters for each service: required $E_{b} / N_{0}(\mathrm{~dB})$; activity factor, $\delta$; and process gain, $G_{p}$. This value of optimum $M_{S H}$ provided a capacity gain of $18-21 \%$ and an increase of required resources of nearly $70 \%$. Therefore, the SSDT gain was not substantially affected by the offered service. The $M_{S H}$ could be maintained below $3-4 \mathrm{~dB}$ (capacity gain around $15 \%$ ) in order to reduce the allocated resources (increased around $40 \%$ ).

Fig. 6 shows the results for combined multimedia mobile services with the following profile: $80 \%$ voice users, $15 \% 144 \mathrm{~Kb} / \mathrm{s}$ data users and $5 \% 384 \mathrm{~Kb} / \mathrm{s}$ data users. The increase percentages of the capacity and required resources are similar to those shown in Fig. 5. The optimum $M_{S H}$ was around $5 \mathrm{~dB}$, the capacity was increased $18 \%$ and the number of resources for $M_{S H}=5 \mathrm{~dB}$ increased $64 \%$. Thus, the optimum $M_{S H}$ and the increase of capacity were not affected substantially by the combination of services.

In Fig. 7, where the cell radius was varied from 300 to 700 meters, it is shown that the higher the radius is, the lower the system capacity and SSDT gain are. However, the optimum $M_{S H}$ ranged from 5 to $5.5 \mathrm{~dB}$. This result could be applied to system planning, where the SSDT gain allows an increase in the cell radius while maintaining the number of users served by simply adjusting the $M_{S H}$ to the appropriate value. 
The factor that mainly affects Soft Handover behavior in a WCDMA system is the propagation scenario. There are three parameters that characterize the propagation scenario: the shadowing typical deviation, $\sigma$, the path loss exponent, $\mu$, and the correlation coefficient between the shadowing from two base stations, $\rho$.

In Fig. 8, the optimum $M_{S H}$ is plotted versus shadowing typical deviation. When $\sigma$ was varied from 0 to $12 \mathrm{~dB}$, the optimum $M_{S H}$ increased from 0 to $7 \mathrm{~dB}$, which is a wide variation depending on the environment conditions. Furthermore, Fig. 9 shows that the capacity gain increased as the shadowing typical deviation increased.

The results obtained for the shadowing correlation $(\rho)$ were similar: the lower the correlation coefficient (defined between -1 and 1) was, the higher the SSDT gain was (Fig.10). The optimum $M_{S H}$ ranged from 0 to $8 \mathrm{~dB}$ when $\rho$ was modified from -1 to 1 . Therefore, the SSDT gain ranged from $18 \%$ to $28 \%$ in real scenarios (the correlation coefficient for the shadowing was between 0.2 and $0.5[7])$.

In Fig. 11, the number of served users is plotted for several propagation constant values. System capacity was improved when $\mu$ was increased, due to interference reduction. Under these conditions, the capacity gain given by SSDT and optimum $M_{S H}$ were slightly reduced.

For all the simulations in this study, two user distributions in the area were considered: a uniform user distribution (by default) and a concentration of all the users in the triangle location that requires a maximum transmitted power (the worst case). Fig. 12 shows the comparison between both user distributions. This figure shows that the capacity improvement given by SSDT is similar when the users are located at the worst location as well as when they are uniformly distributed.

\section{CONCLUSIONS}

In this paper, we have analyzed SSDT capacity gain, concluding that the WCDMA system capacity can always be improved by using SSDT technique. The increase in capacity is achieved not only by using SC between the signals from different base stations, but also due to the interference reduction provided by the SSDT SIR-based power control. 
However, this capacity gain leads to an increase in the number of resources used in the radio interface (channelling and scrambling codes) as well as an increase in the transmission network (channel elements, processing signal equipment, radio links, dedicated circuits...). This trade-off between the capacity gain and the increase of required resources can be controlled by the SHO margin $\left(M_{S H}\right)$. The $M_{S H}$ can be fixed to $3-4 \mathrm{~dB}$ to reduce the percentage of resources allocated due to the drastic increase of resources as a function of soft handover margin.

In this study, the system behavior in a macrocellular scenario has been analyzed for different propagation conditions, types of service, user distributions, shadowing correlation coefficients, and cell radii. In all cases, the capacity and dedicated resources increased with the use of SSDT, and the optimal SHO margin was calculated for each situation. From the results of this paper, the types of service or combined services do not substantially affect on the increase in capacity or the optimum soft handover margin. Propagation parameters significantly influence the optimum $M_{S H}$ and the increase in capacity.

\section{APPENDIX}

The connection probabilities can be expressed as function of the bivariate normal probability function defined as [22, eq. (26.3.3)]

$$
L(h, k, \rho)=\frac{1}{2 \pi \sqrt{1-\rho^{2}}} \int_{h}^{\infty} d x \int_{k}^{\infty} \exp \left(-\frac{x^{2}-2 \rho x y+y^{2}}{2\left(1-\rho^{2}\right)}\right) d y
$$

Let the random variables $X, Y$ be distributed as a bivariate normal distribution with means $\left(m_{x}, m_{y}\right)$ and variances $\left(\sigma_{x}^{2}, \sigma_{y}^{2}\right)$ and correlation. The cumulative density probability $(\mathrm{CDF})$ of $X$ and $Y$ is given by

$$
\begin{aligned}
& \operatorname{Prob}(X \leq h, Y \leq k)=\frac{1}{2 \pi \sigma_{x} \sigma_{y} \sqrt{1-\rho^{2}}} \int_{-\infty}^{\frac{h-m_{x}}{\sigma_{x}}} d x \int_{-\infty}^{\frac{k-m_{y}}{\sigma_{y}}} \exp \left(-\frac{x^{2}-2 \rho x y+y^{2}}{2\left(1-\rho^{2}\right)}\right) d y= \\
& L\left(-\left(\frac{h-m_{x}}{\sigma_{x}}\right),-\left(\frac{k-m_{y}}{\sigma_{y}}\right), \rho\right)
\end{aligned}
$$

A. Properties of $L(h, k, \rho)$

$$
\begin{aligned}
& L(h, k, \rho)=L(k, h, \rho) \\
& L(-h,-k, \rho)-L(h, k, \rho)=1-Q(k)-Q(h)
\end{aligned}
$$


B. Examples of Probability Calculations

1) Calculation of $\operatorname{Prob}\left(P_{r_{1, l}}-P_{r_{2, l}}>M_{S H}, P_{r_{2, l}}-P_{r_{3, l}} \geq 0\right)$

Assume the Gaussian random variables $P_{r_{1, l}}, P_{r_{2, l}}$ and $P_{r_{3, l}}$ which correspond to (1) expressed in logarithmic units, following a constant correlation model with $\rho_{i j}=\rho, i, j=1,2,3$, $i \neq j$.

Let $s_{1}$ and $s_{2}$ be defined as

$$
s_{1}=P_{r_{1, l}}-P_{r_{2, l}} \quad s_{2}=P_{r_{2, l}}-P_{r_{3, l}} .
$$

The correlation coefficient between $s_{1}$ and $s_{2}, \rho_{s_{12}}$, can be written as

$$
\rho_{s_{12}}=\frac{E\left[s_{1} \cdot s_{2}\right]-E\left[s_{1}\right] \cdot E\left[s_{2}\right]}{\sigma_{e q}^{2}},
$$

where $\sigma_{e q}$ is given by (13).

Substituting (44) into (45), it yields

$$
\rho_{s_{12}}=\frac{E\left[\left(P_{r_{1, l}}-P_{r_{2, l}}\right) \cdot\left(P_{r_{2, l}}-P_{r_{3, l}}\right)\right]-E\left[\left(P_{r_{1, l}}-P_{r_{2, l}}\right)\right] \cdot E\left[\left(P_{r_{2, l}}-P_{r_{3, l}}\right)\right]}{\sigma_{e q}^{2}} .
$$

Since $\rho$ is defined as $(3)$

$$
\begin{aligned}
& \rho=\frac{E\left[P_{r_{1, l}} \cdot P_{r_{2, l}}\right]-E\left[P_{r_{1, l}}\right] \cdot E\left[P_{r_{2, l}}\right]}{\sigma^{2}}=\frac{E\left[P_{r_{1, l}} \cdot P_{r_{3, l}}\right]-E\left[P_{r_{1, l}}\right] \cdot E\left[P_{r_{3, l}}\right]}{\sigma^{2}}= \\
& =\frac{E\left[P_{r_{2, l}} \cdot P_{r_{3, l}}\right]-E\left[P_{r_{2, l}}\right] \cdot E\left[P_{r_{3, l}}\right]}{\sigma^{2}},
\end{aligned}
$$

from (46) and (47), we can obtain $\rho_{s_{12}}=-1 / 2$

The probability $\operatorname{Prob}\left(P_{r_{1, l}}-P_{r_{2, l}}>M_{S H}, P_{r_{2, l}}-P_{r_{3, l}} \geq 0\right)$ can be evaluated using (40) and (41) as

$$
\operatorname{Prob}\left(P_{r_{1, l}}-P_{r_{2, l}}>M_{S H}, P_{r_{2, l}}-P_{r_{3, l}} \geq 0\right)=L\left(\frac{M_{S H}-\overline{s_{1}}}{\sigma_{e q}}, \frac{-\overline{s_{2}}}{\sigma_{e q}},-\frac{1}{2}\right)
$$

From (1) and (44), we can calculate (48) as 
$\operatorname{Prob}\left(P_{r_{1, l}}-P_{r_{2, l}}>M_{S H}, P_{r_{2, l}}-P_{r_{3, l}} \geq 0\right)=L\left(\frac{M_{S H}-10 \mu \log \left(\frac{d_{2, l}}{d_{1, l}}\right)}{\sigma_{e q}}, \frac{10 \mu \log \left(\frac{d_{2, l}}{d_{3, l}}\right)}{\sigma_{e q}},-\frac{1}{2}\right)$

2) Calculation of $\operatorname{Prob}\left(0 \leq P_{r_{1, l}}-P_{r_{2, l}}<M_{S H}, 0 \leq P_{r_{1, l}}-P_{r_{3, l}}<M_{S H}\right)$

This probability can be reduced to

$\operatorname{Prob}\left(0 \leq P_{r_{1, l}}-P_{r_{2, l}}<M_{S H}, 0 \leq P_{r_{1, l}}-P_{r_{3, l}}<M_{S H}\right)=\operatorname{Prob}\left(P_{r_{1, l}}-P_{r_{2, l}} \geq 0, P_{r_{1, l}}-P_{r_{3, l}} \geq 0\right)-$

$-\operatorname{Prob}\left(P_{r_{1, l}}-P_{r_{2, l}}>M_{S H}, P_{r_{1, l}}-P_{r_{3, l}}>0\right)-\operatorname{Prob}\left(P_{r_{1, l}}-P_{r_{2, l}}>0, P_{r_{1, l}}-P_{r_{3, l}}>M_{S H}\right)+\quad$.

$+\operatorname{Prob}\left(P_{r_{1, l}}-P_{r_{2, l}} \geq M_{S H}, P_{r_{1, l}}-P_{r_{3, l}} \geq M_{S H}\right)$

Using a procedure that is analogous to (44)-(49), we can evaluate the probabilities involved in (50) as

$\operatorname{Prob}\left(0 \leq P_{r_{1, l}}-P_{r_{2, l}}<M_{S H}, 0 \leq P_{r_{1, l}}-P_{r_{3, l}}<M_{S H}\right)=L\left(\frac{10 \mu \log \left(\frac{d_{1, l}}{d_{2, l}}\right)}{\sigma_{e q}}, \frac{10 \mu \log \left(\frac{d_{1, l}}{d_{3, l}}\right)}{\sigma_{e q}}, \frac{1}{2}\right)-$
$-L\left(\frac{M_{S H}+10 \mu \log \left(\frac{d_{1, l}}{d_{2, l}}\right)}{\sigma_{e q}}, \frac{10 \mu \log \left(\frac{d_{1, l}}{d_{3, l}}\right)}{\sigma_{e q}}, \frac{1}{2}\right)-L\left(\frac{10 \mu \log \left(\frac{d_{1, l}}{d_{2, l}}\right)}{\sigma_{e q}}, \frac{M_{S H}+10 \mu \log \left(\frac{d_{1, l}}{d_{3, l}}\right)}{\sigma_{e q}}, \frac{1}{2}\right)+$
$+L\left(\frac{M_{S H}+10 \mu \log \left(\frac{d_{1, l}}{d_{2, l}}\right)}{\sigma_{e q}}, \frac{M_{S H}+10 \mu \log \left(\frac{d_{1, l}}{d_{3, l}}\right)}{\sigma_{e q}}, \frac{1}{2}\right)$

\section{ACKNOWLEDGMENT}

The author would like to thank L. Bueno and D. Romero for their simulation programs and P. Guerediaga, J.A. Pons and J.C. del Rio for their technical support. Furthermore, the author expresses his gratitude to Dr. N. Cardona for his revision of the text. Finally, J. Reig would like to thank the Editor and the anonymous reviewers for their contributions to enrich the final paper. 


\section{REFERENCES}

[1] H. Holma and A. Toskala, WCDMA for UMTS: Radio Access for Third Generation Mobile Communications. New York: John Wiley \& Sons, 2001.

[2] D. Wong and T. J. Lim, "Soft handoffs in CDMA mobile systems," IEEE Pers. Commun., vol. 4, pp. 6-17, Dec. 1997.

[3] Radio Resource Control (RRC); Protocol Specification, 3GPP TSG RAN 25.331 V.5.2.0, 2002.

[4] Physical Layer Procedures (FDD), 3GPP TSG RAN 25.214 V.5.2.0, 2002.

[5] A. J. Viterbi, A. M. Viterbi, K. S. Gilhousen and E. Zehavi, "Soft handoff extends CDMA cell coverage and increases reverse link capacity," IEEE J. Select. Areas Commun., vol. 12, pp. 1281-1288, Oct. 1994.

[6] C. Mehailescu, X. Lagrange and Ph. Godlewski, "Soft handover analysis in downlink UMTS WCDMA system," in Proc. of IEEE MoMuC, San Diego, 1999, pp. 279-285.

[7] X. Yang, S. Ghaheri-Niri and R. G Tafazolli, "Downlink soft handover gain in CDMA cellular network with cross-correlated shadowing," in Proc. IEEE Vehicular Technology Conf. VTC 2001 Fall, Oct. 2001, vol. 1, pp. 276-280.

[8] D. Zhang, G. Wei and J. Zhu, "Performance of hard and soft handover for CDMA system," in Proc. IEEE Vehicular Technology Conf. VTC 2002 Fall, Oct. 2002, vol. 2, pp. 1143-1147.

[9] Y. Chen and L. Cuthbert, "Optimum size of soft handover zone in power-controlled UMTS downlink systems," Electron. Lett., vol. 38, pp. 89-90, Jan. 2002.

[10] D. Staehle, K. Leibnitz and K. Heck, "Effects of soft handover on the UMTS downlink performance," in Proc. IEEE Vehicular Technology Conf. VTC 2002 Fall, Oct. 2002, vol.2, pp. 960-964.

[11] K. Heck, D. Staehle and K. Leibnitz, "Diversity effects of the soft handover gain in UMTS downlink networks," in Proc. IEEE Vehicular Technology Conf. VTC 2002 Fall, Oct. 2002, vol. 2, pp. 1269-1273. 
[12] Y. P. E. Wang and G. E. Bottomley, "CDMA downlink system capacity enhancement through generalized RAKE reception," in Proc. IEEE Vehicular Technology Conf. VTC 2001 Fall, Oct. 2001, vol. 2, pp. 1177-1181.

[13] S. Akhtar, S. A. Malik and D. Zeghlache, "A comparative study of power control strategies for soft handover in UTRA FDD WCDMA system," in Proc. IEEE Vehicular Technology Conf. VTC 2001 Spring, May 2001, vol. 4, pp. 2680-2684.

[14] A. Marimoto, K. Higuchi and M. Sawahashi, "Site independent diversity transmit power control for inter-cell diversity in W-CDMA forward link," in Proc. IEEE Vehicular Technology Conf. VTC 2001 Fall, Oct. 2001, pp. 645-649.

[15] N. Takano and K. Hamabe, "Enhancement of site selection diversity transmit power control in CDMA cellular systems," in Proc. IEEE Vehicular Technology Conf. VTC-2001 Fall, Oct. 2001, pp. 635-639.

[16] H. Furukawa, K. Hamabe and A. Ushirokawa, "SSDT-site selection diversity transmission power control for CDMA forward link," IEEE J. Select. Areas Commun., vol. 18, pp. 15461553, Aug. 2000.

[17] J. D. Parsons, The Mobile Radio Propagation Channel. London: Pentech-Press Publishers, 2000.

[18] A. J. Viterbi, A. M. Viterbi and E. Zehavi, "Other cell interference in cellular powercontrolled CDMA," IEEE Trans. Commun., vol. 42, pp. 1501-1504, Apr. 1994.

[19] A. Papoulis, Probability, Random Variables, and Stochastic Processes. Singapore: McGrawHill, 1984.

[20] S. Hämäläinen, H. Holma A. Toskala and M. Laukkanen, "Analysis of CDMA downlink capacity enhancements," in Proc. of PIMRC Symposium, 1997, pp. 241-245.

[21] A. Viterbi, CDMA Principles of Spread Spectrum Communication. New York: AddisonWesley, 1996.

[22] M. Abramowitz and I. Stegun, Handbook of Mathematical Functions. Washington: National Bureau of Standards, 1972. 
FIGURES AND TABLES 


\begin{tabular}{|c|c|c|c|c|}
\hline Parameters & Voice & $144 \mathrm{~Kb} / \mathrm{s}$ data & $384 \mathrm{~Kb} / \mathrm{s}$ data & $2 \mathrm{Mb} / \mathrm{s}$ data \\
\hline $\begin{array}{l}\text { Required } E_{b} / N_{0} \\
(\mathrm{~dB})\end{array}$ & 6.4 & 0.8 & 1.4 & 1.4 \\
\hline $\begin{array}{l}\text { Activity factor } \\
\delta\end{array}$ & 0.5 & 0.4 & 0.4 & 0.4 \\
\hline $\begin{array}{l}\text { Process gain } \\
G_{p}\end{array}$ & 314.75 & 26.66 & 10 & 1.92 \\
\hline
\end{tabular}

Table 1. Parameters used in the simulations 


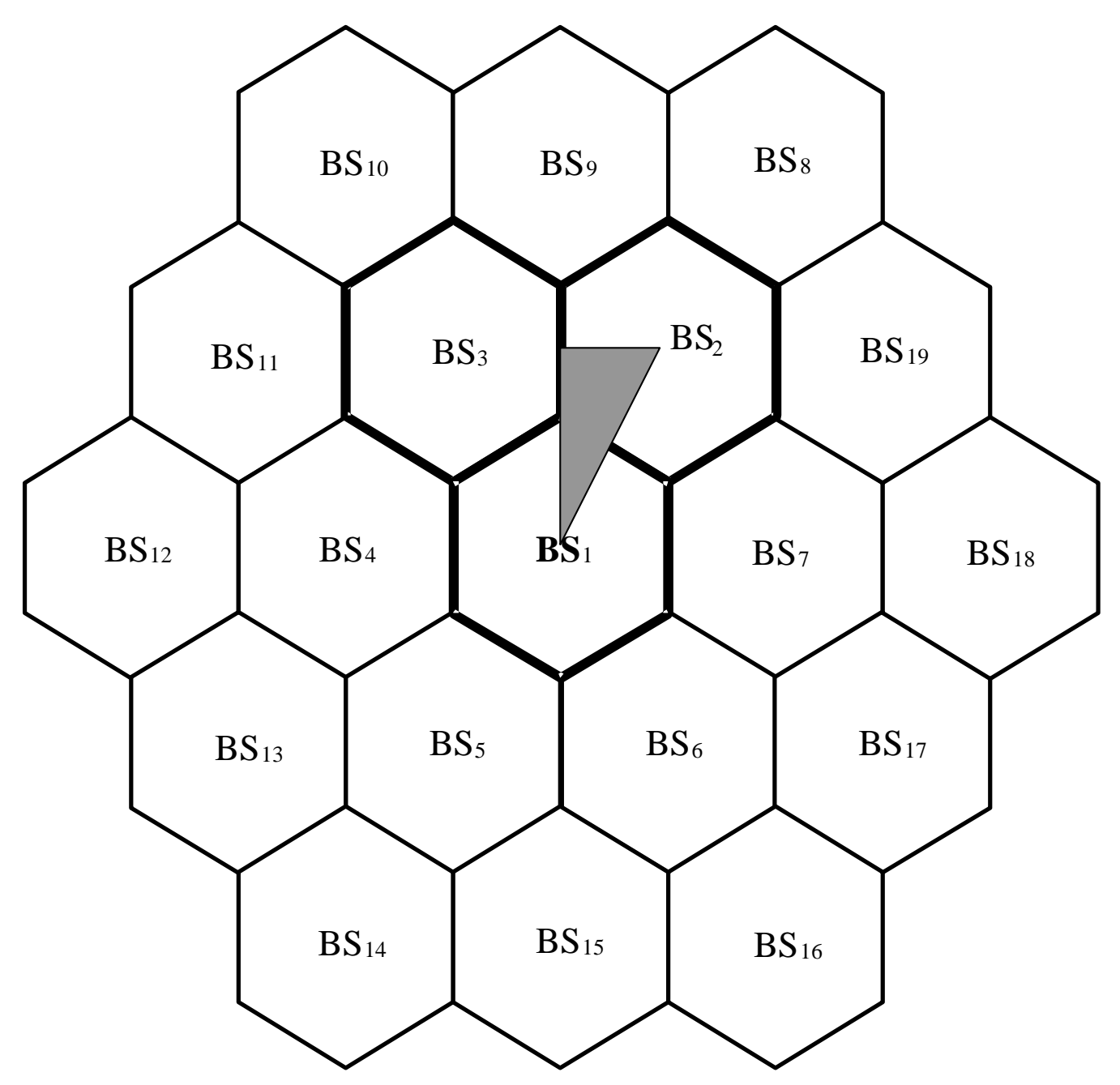

Fig. 1. Cellular scenario considered for the analysis 

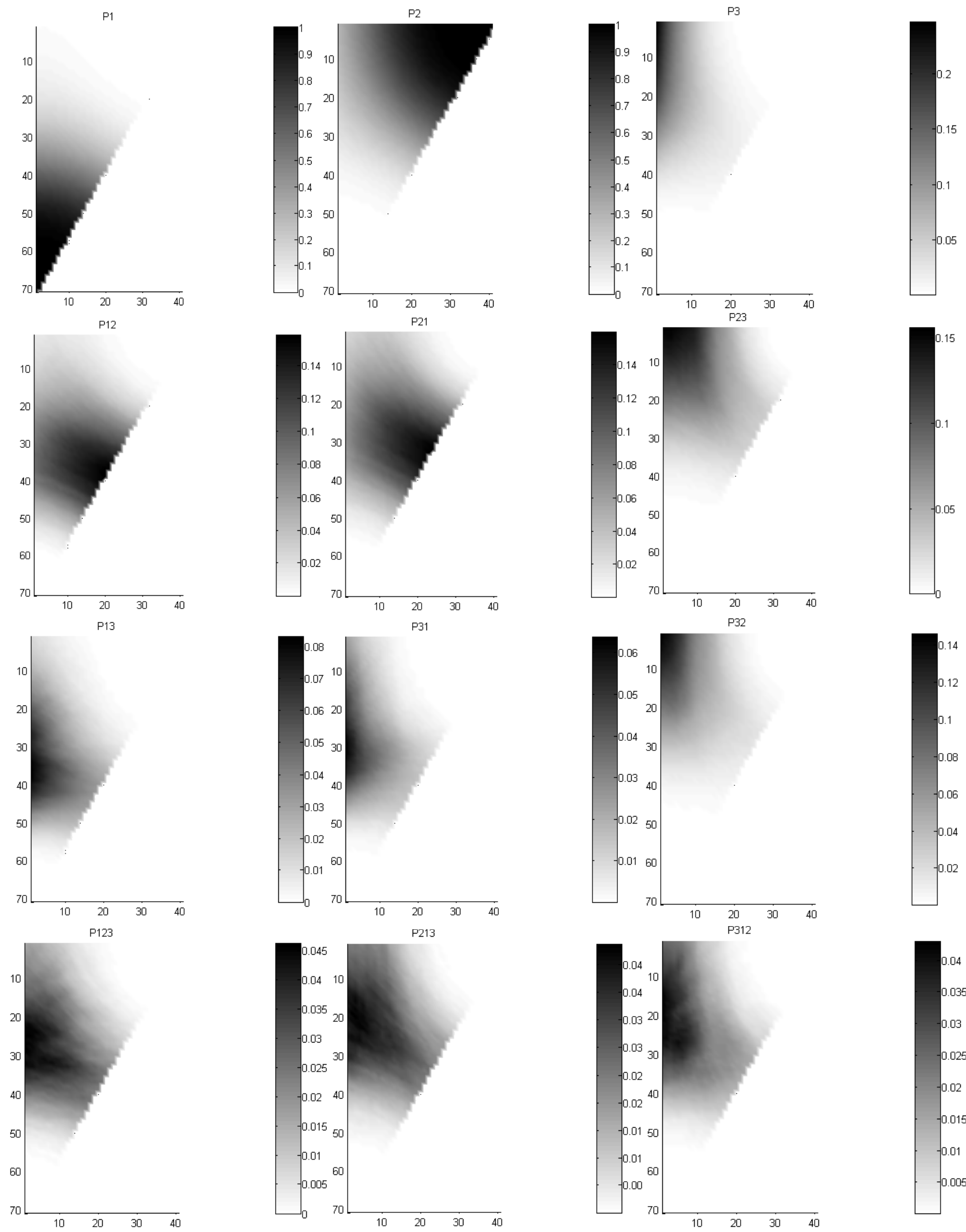

Fig. 2. Connection probabilities for $M_{S H}=4 \mathrm{~dB}, \sigma=8 \mathrm{~dB}, \rho=0.5$ 


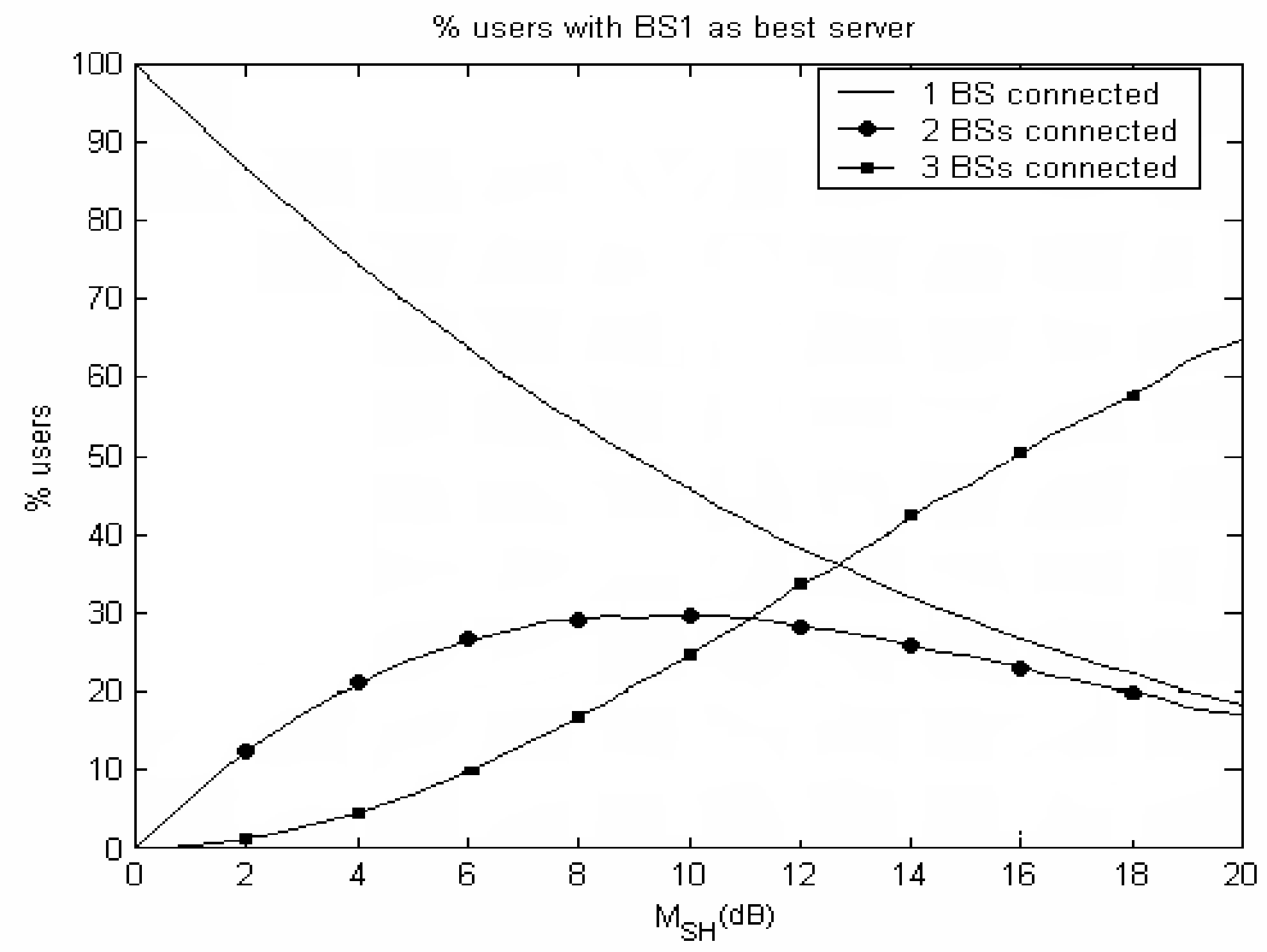

Fig. 3. Percentage of users connected to 1, 2 or 3 base stations as a function of the soft handover margin $\left(M_{S H}\right)$, with $\mathrm{BS}_{1}$ being the best server. 


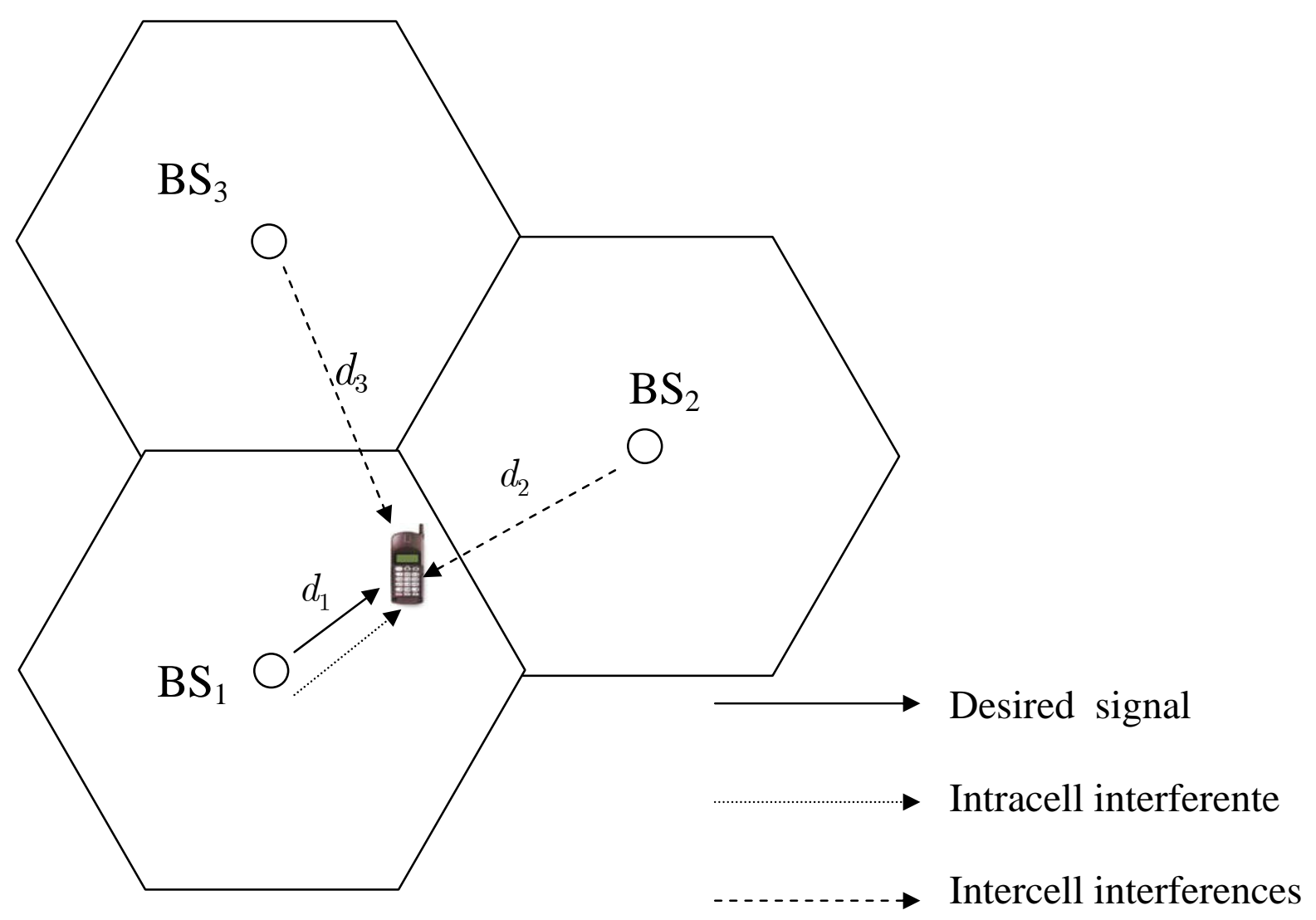

Fig. 4. Signals received in the mobile stations 


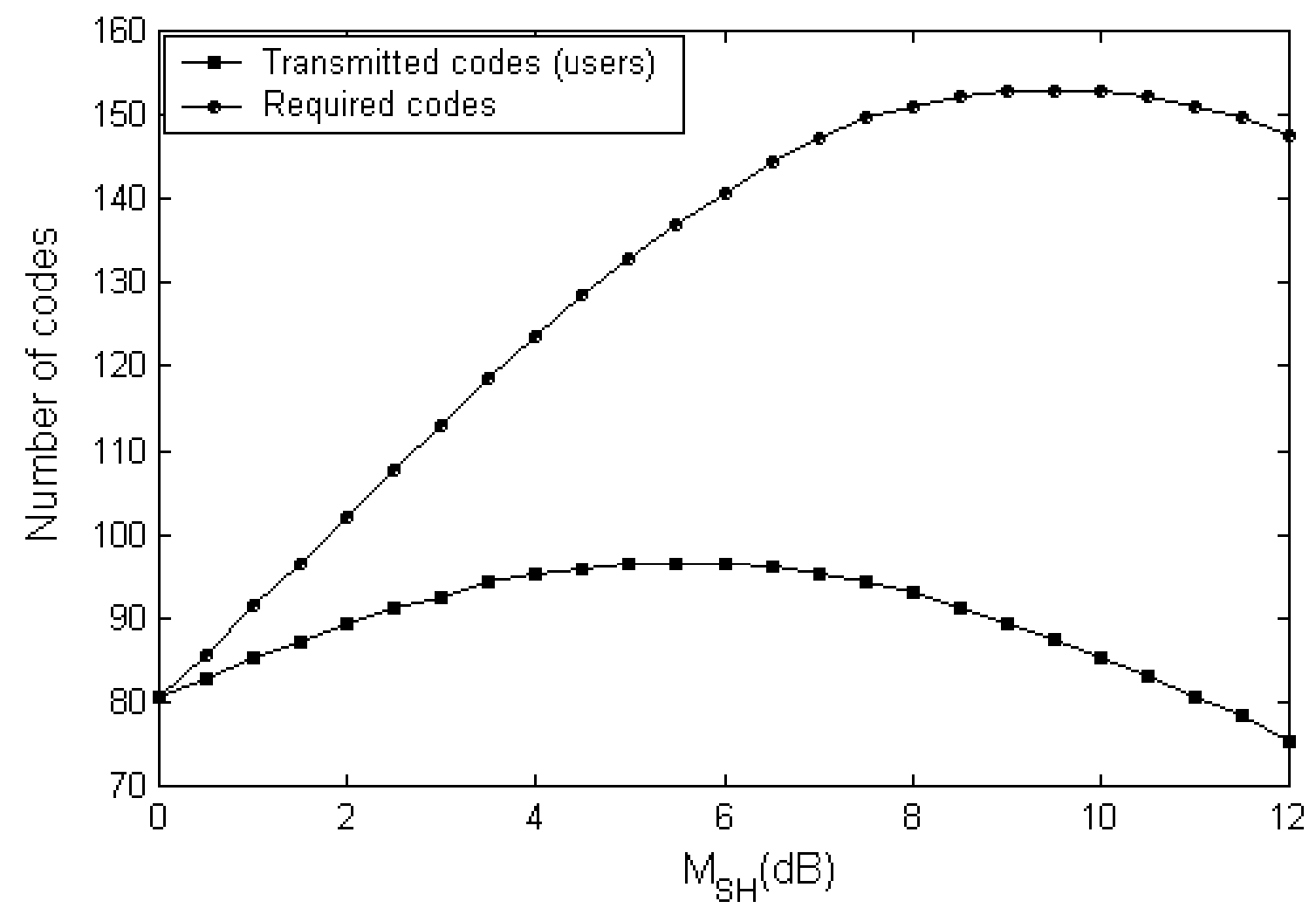

Fig. 5. Required and transmitted codes (number of users) by $\mathrm{BS}_{1}$ versus soft handover margin, $M_{S H}(\mathrm{~dB})$ for a uniform distribution of users. 


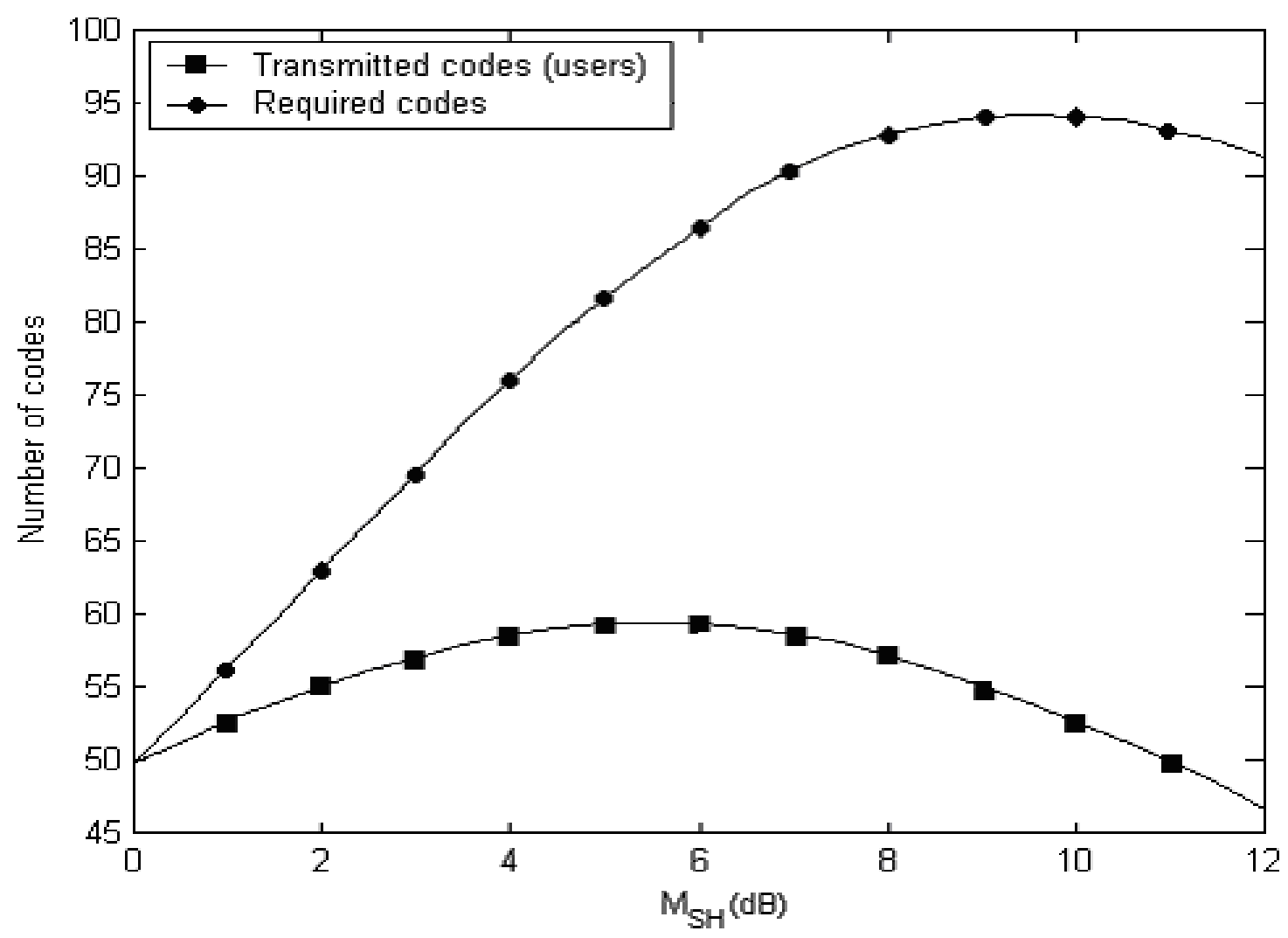

Fig. 6. Required and transmitted codes (number of users) by $\mathrm{BS}_{1}$ versus soft handover margin, $M_{S H}(\mathrm{~dB})$ for a uniform distribution of users. Services distribution: $80 \%$ voice, $15 \% 144 \mathrm{~Kb} / \mathrm{s}$ data and $5 \% 384 \mathrm{~Kb} / \mathrm{s}$ data. 


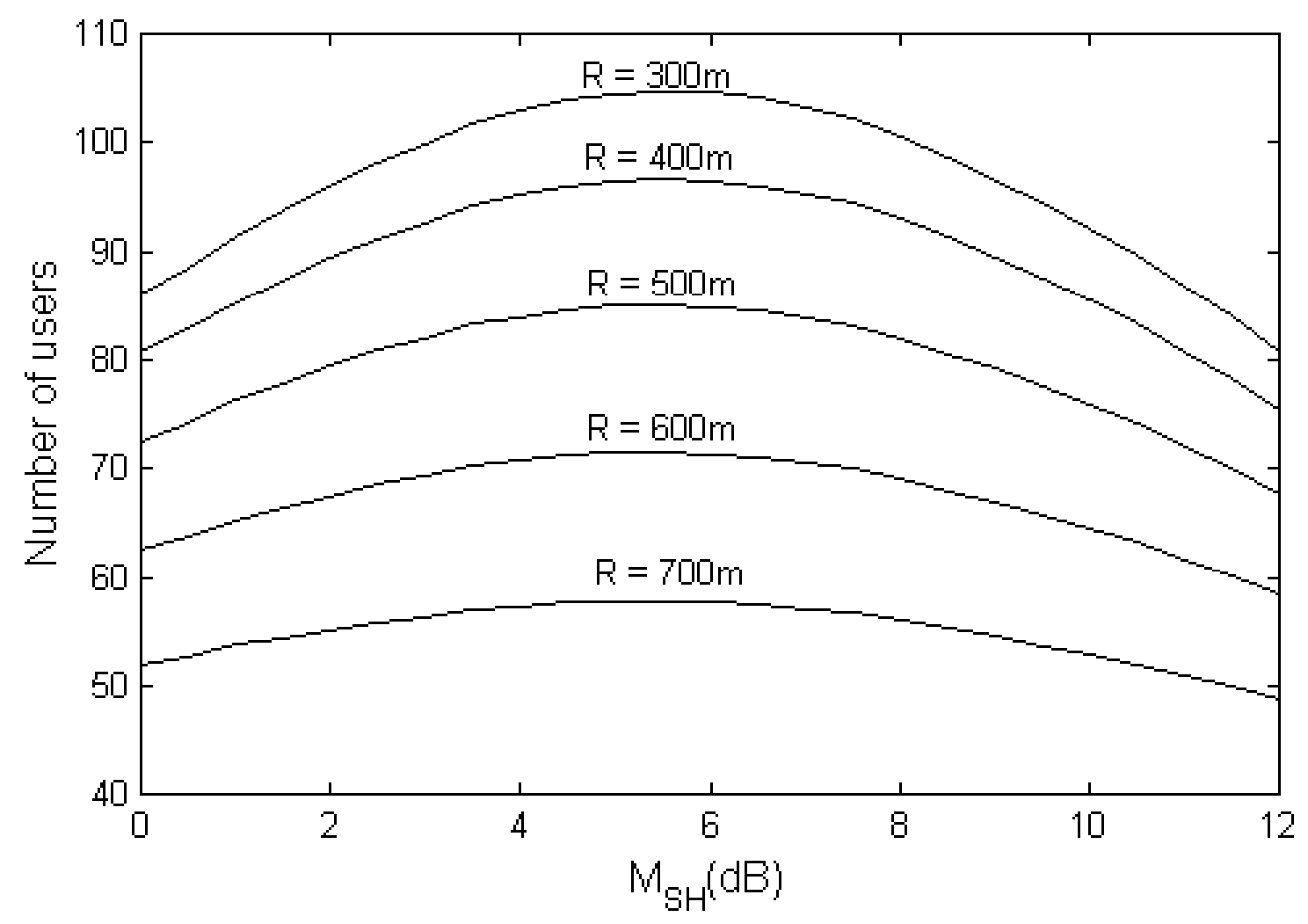

Fig. 7. System capacity vs. soft handover margin, $M_{S H}(\mathrm{~dB})$, for different cell radii. 


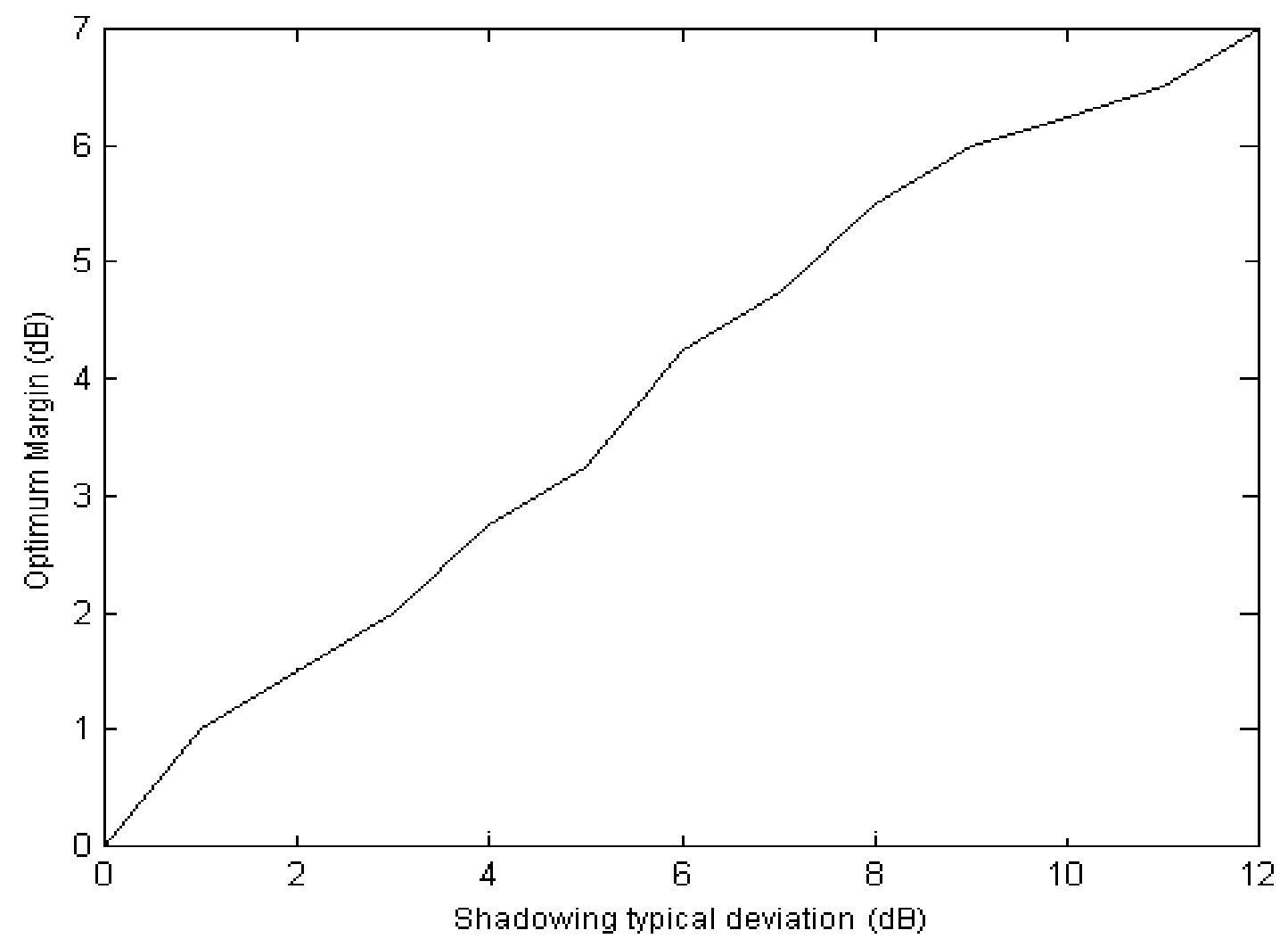

Fig. 8. Optimum soft handover margin, $M_{S H}$, as a function of shadowing standard deviation, $\sigma(\mathrm{dB})$. 


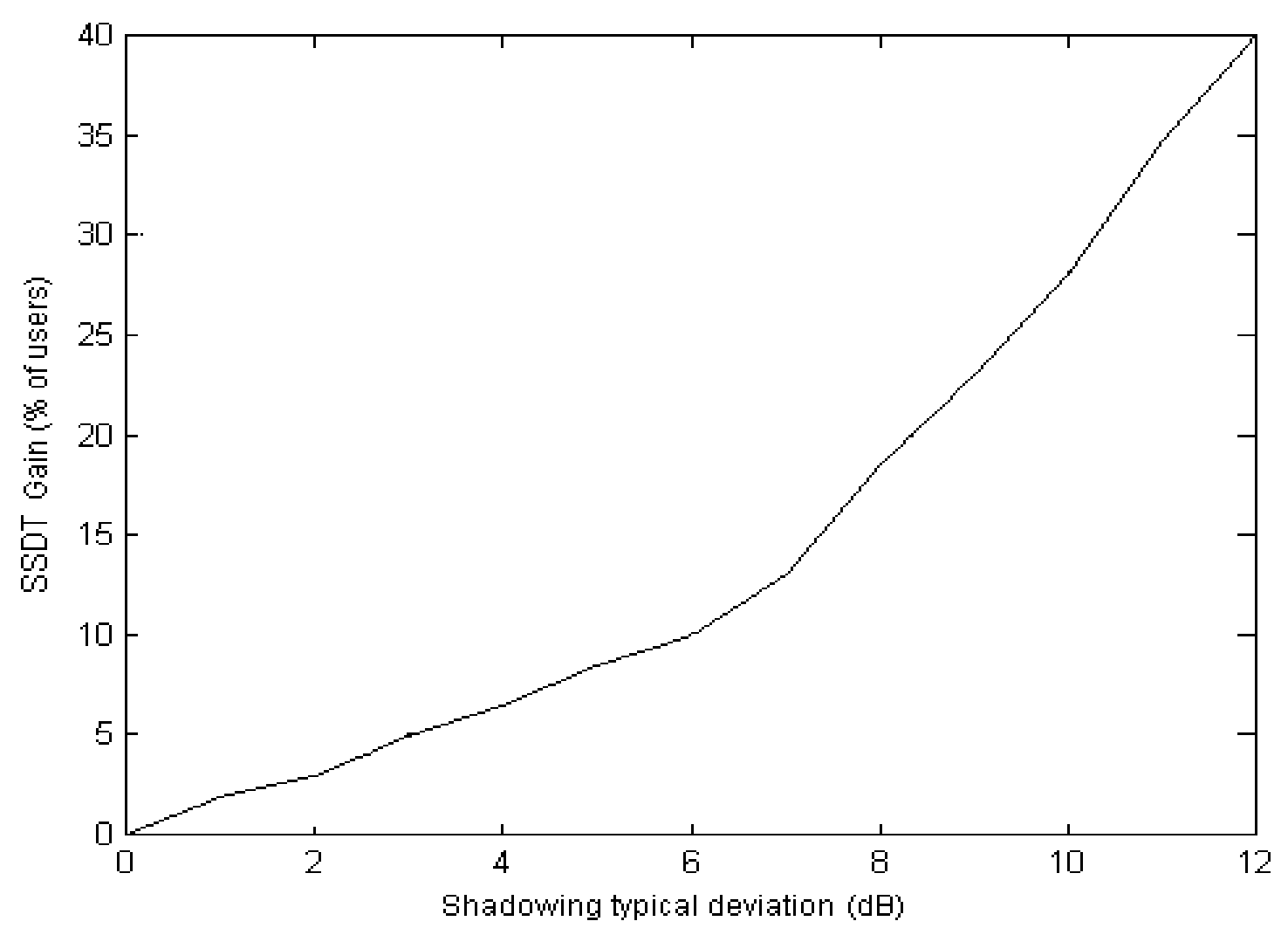

Fig. 9. SSDT capacity gain as a function of the shadowing standard deviation, $\sigma(\mathrm{dB})$. 


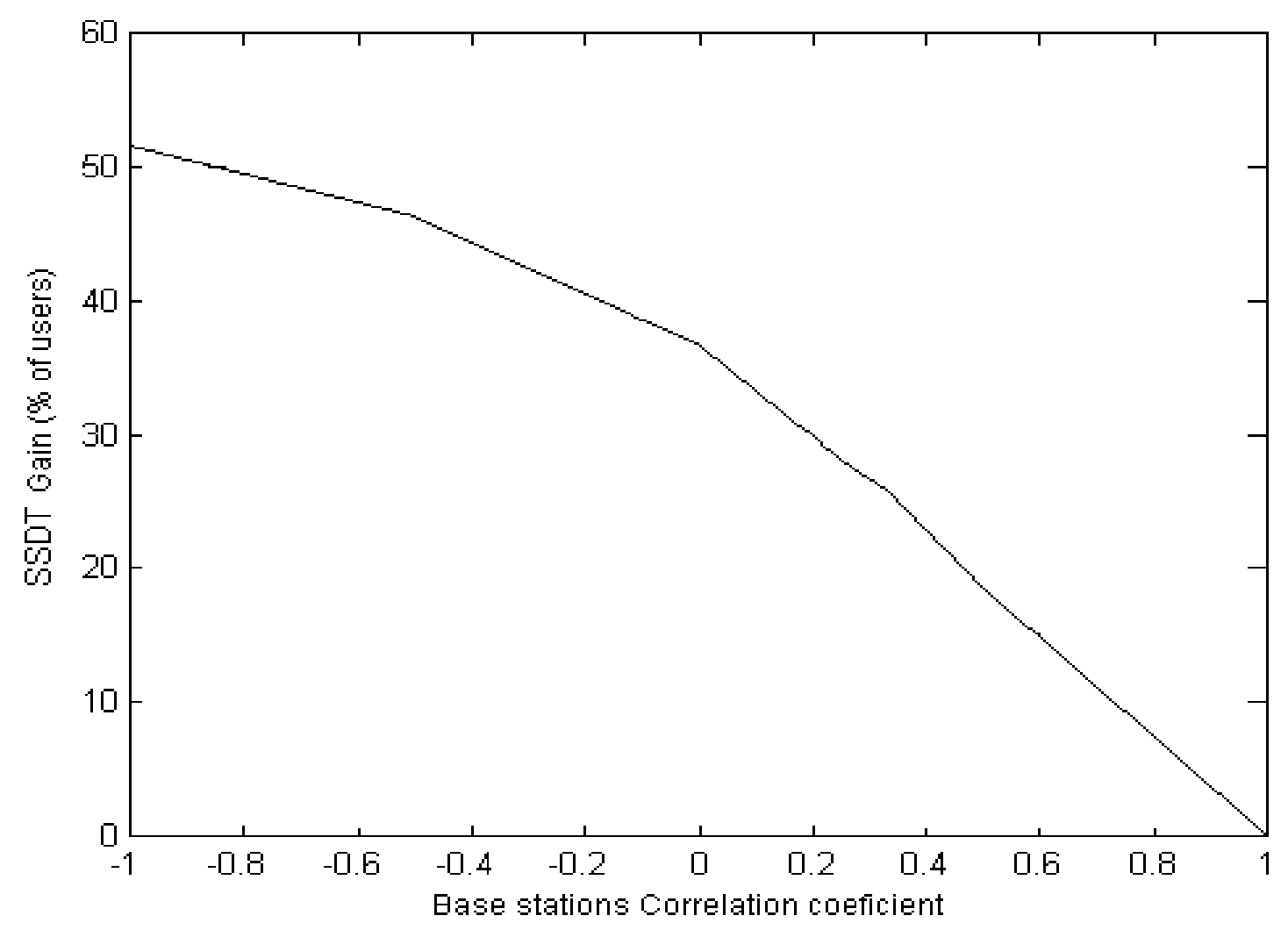

Fig. 10. SSDT capacity gain as a function of the correlation coefficient between shadowing, $\rho$. 


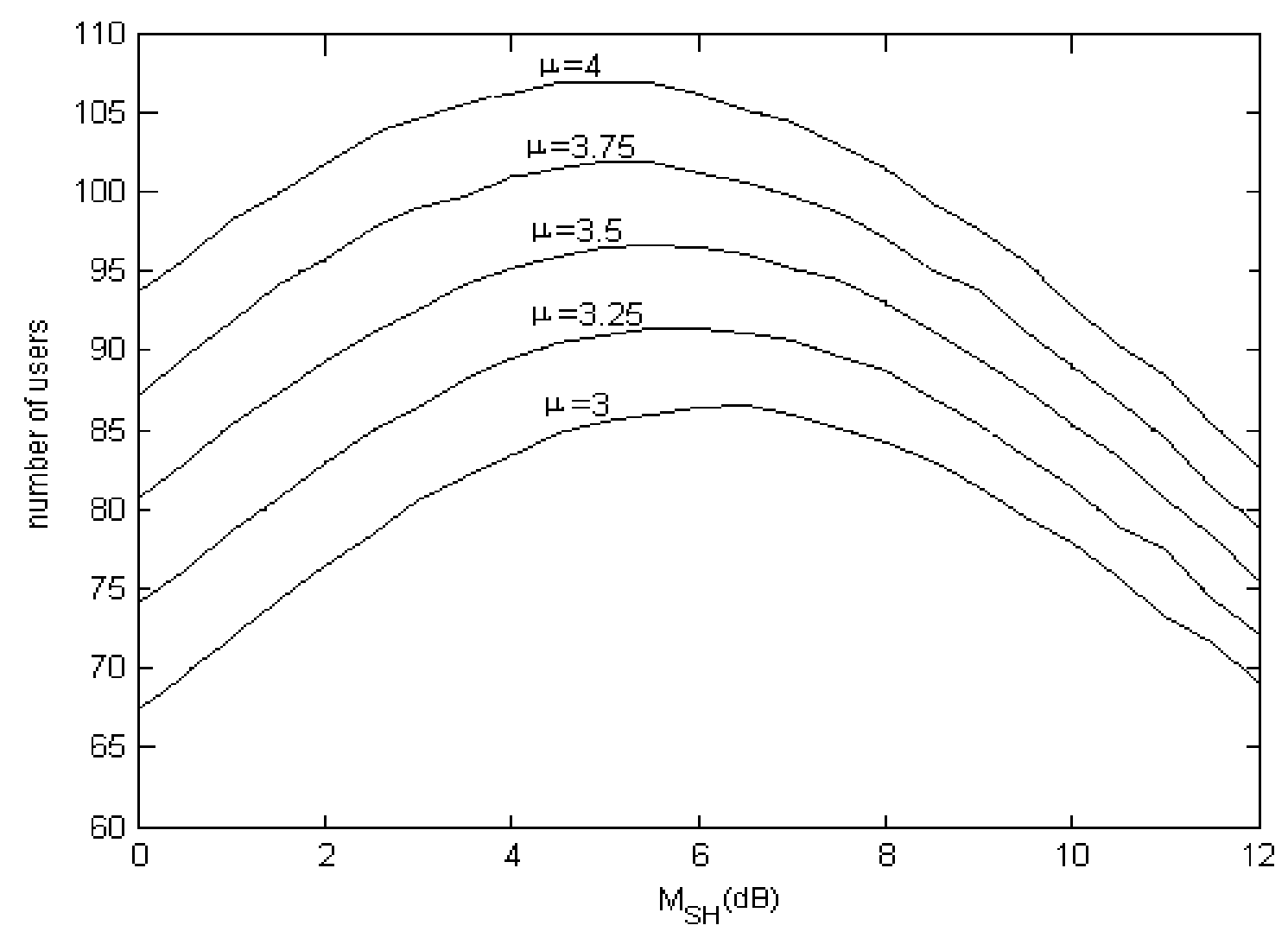

Fig. 11. System capacity as a function of the soft handover margin, $M_{S H}(\mathrm{~dB})$, for different path loss exponents. 


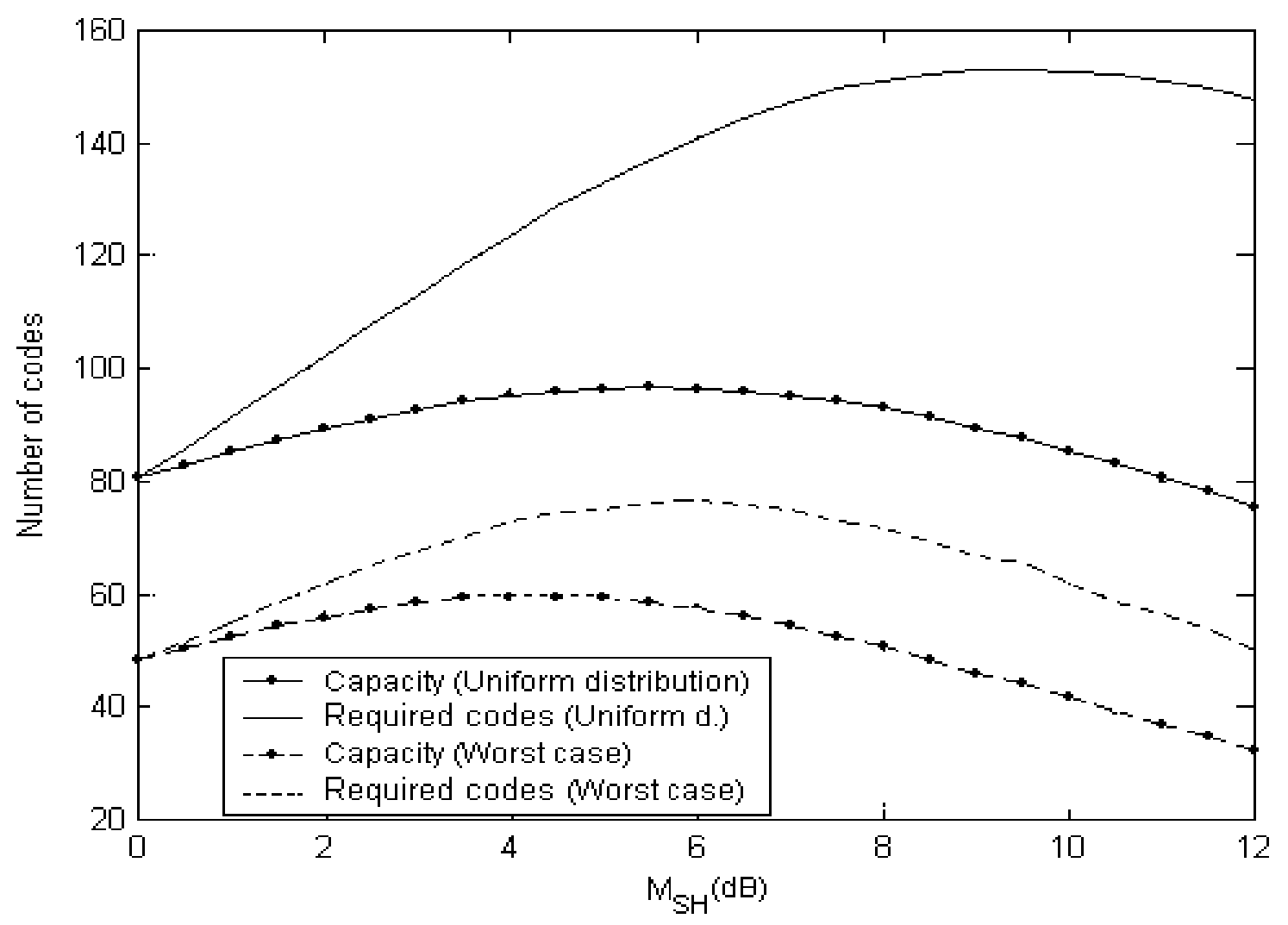

Fig. 12. Capacity and required codes by base station for a uniform distribution and for the worst case (all the users located in the point of the maximum required power from the base station). 